\title{
Managing radiation degradation of CCDs on the Chandra X-ray Observatory-III
}

\author{
Stephen L. O'Dell ${ }^{*}{ }^{\mathrm{a}}$, Thomas L. Aldcroft ${ }^{\mathrm{b}}$, William C. Blackwell ${ }^{\mathrm{c}}$, Sabina L. Bucher ${ }^{\mathrm{d}}$, \\ Jon H. Chappell ${ }^{\mathrm{b}}$, Joseph M. DePasquale ${ }^{\mathrm{b}}$, Catherine E. Grant ${ }^{\mathrm{e}}$, Michael Juda ${ }^{\mathrm{b}}$, Eric R. Martin ${ }^{\mathrm{c}}$, \\ Joseph I. Minow, Stephen S. Murray ${ }^{\mathrm{b}}$, Paul P. Plucinsky, Daniel A. Schwartz, \\ Daniel P. Shropshire ${ }^{c}$, Bradley J. Spitzbart ${ }^{b}$, Paul R. Viens ${ }^{c}$, and Scott J. Wolk ${ }^{b}$
${ }^{a}$ NASA Marshall Space Flight Center, NSSTC/VP62, 320 Sparkman Dr., Huntsville, AL 35805
${ }^{b}$ Harvard-Smithsonian Center for Astrophysics, 60 Garden St., Cambridge, MA 02138
${ }^{c}$ Jacobs Sverdrup, Sverdrup/MSFC, P.O. Box 9030, Huntsville, AL 35812
${ }^{\mathrm{e}}$ MIT Center for Space Research, MIT, 77 Massachusetts Ave., Cambridge, MA 02139
${ }^{f}$ NASA Marshall Space Flight Center, MSFC/EV13, Huntsville, AL 35812 \\ ${ }^{d}$ Northrop Grumman Space Technology, CfA/MS33, 60 Garden St., Cambridge, MA 02138
}

\begin{abstract}
The CCDs on the Chandra X-ray Observatory are vulnerable to radiation damage from low-energy protons scattered off the telescope's mirrors onto the focal plane. Following unexpected damage incurred early in the mission, the Chandra team developed, implemented, and maintains a radiation-protection program. This program-involving scheduled radiation safing during radiation-belt passes, intervention based upon real-time space-weather conditions and radiationenvironment modeling, and on-board radiation monitoring with autonomous radiation safing-has successfully managed the radiation damage to the CCDs. Since implementing the program, the charge-transfer inefficiency (CTI) has increased at an average annual rate of only $3.2 \times 10^{-6}(2.3 \%)$ for the front-illuminated CCDs and $1.0 \times 10^{-6}(6.7 \%)$ for the back-illuminated CCDs. This paper describes the current status of the Chandra radiation-management program, emphasizing enhancements implemented since the previous papers.
\end{abstract}

Keywords: X-ray astronomy, CCDs, radiation damage, radiation environment, spacecraft operations

\section{INTRODUCTION}

Now 8 years into its mission, the Chandra $X$-ray $O b$ servatory $^{1,2,3}$ continues to provide superb arcsecond imaging, imaging spectrometry, and high-resolution dispersive spectroscopy. Chandra's Science Instrument Module (SIM) houses 2 instruments, each with an imaging (I) array and with a spectroscopy (S) array for reading spectra dispersed by transmission gratings. The High-Resolution Camera ${ }^{4}$ (HRC) uses microchannel plates. The Advanced CCD Imaging Spectrometer $^{5}$ (ACIS) has 10 CCDs: ACIS-I comprises a $2 \times 2$ CCD array; ACIS-S, a $6 \times 1$ CCD array. Of the 10 CCDs, 2 CCDs - both in the S array - are back-illuminated (BI); the remaining $8 \mathrm{CCDs}$ are front-illuminated (FI).

Upon initial Chandra operations, the ACIS FI CCDs experienced rapid degradation ${ }^{6}$ - a significant increase in chargetransfer inefficiency (CTI). While the CTI of FI CCDs increased, the CTI of BI CCDs did not; nor did the dark current of either device increase: These symptoms indicate that weakly penetrating, low-energy $(0.1-0.5-\mathrm{MeV})$ protons caused this damage. ${ }^{7}$ An urgent anomaly investigation concluded that trapped protons ${ }^{8}$ had Rutherford scattered off $C h a n d r a^{\prime} s$ $\mathrm{x}$-ray mirrors ${ }^{9}$ onto the CCDs during the 8 radiation-belt passages that ACIS had been in the focal position.

Fortunately, upon recognition of this unforeseen problem, the Chandra team immediately modified operating procedures to ensure that the ACIS never remained in the focal position during radiation-belt passes. This policy soon became the keystone to a radiation-protection program ${ }^{10}$ (Paper I) that has successfully limited subsequent radiation damage to acceptable levels. Here we report the status $(\$ 2)$ of that program and describe recent enhancements $(\S 3)$.

\footnotetext{
* Contact author: Steve.O'Dell@nasa.gov; phone 1 256-961-7776; fax 1 256-961-7213; wwwastro.msfc.nasa.gov
} 


\section{STATUS}

Here we discuss Chandra's orbit and radiation environment $(\$ 2.1)$ and the Chandra radiation-protection program (§2.2). With this program in place, increases in the CTI of ACIS CCDs have remained within budgeted levels (\$2.4).

\subsection{Orbit and radiation environment}

The Chandra X-ray Observatory is in a highly elliptical orbit, with a 63.5 -h period and $80.8-\mathrm{Mm}\left(12.7-\mathrm{R}_{\oplus}\right)$ semi-major axis. In the 6 years since reaching operational orbit, Chandra's orbital eccentricity and inclination have evolved from 0.800 and $28.5^{\circ}$ to 0.563 and $57.5^{\circ}$, respectively. Therefore, Chandra's apogee and perigee altitudes have changed from $139 \mathrm{Mm}$ and $10 \mathrm{Mm}\left(22.8 \mathrm{R}_{\oplus}\right.$ and $2.5 \mathrm{R}_{\oplus}$, geocentric) to $120 \mathrm{Mm}$ and $29 \mathrm{Mm}\left(19.8 \mathrm{R}_{\oplus}\right.$ and $5.5 \mathrm{R}_{\oplus}$, geocentric), respectively. Figure 1 shows the evolution of the orbit's inclination, apogee, and perigee, over 25 years.

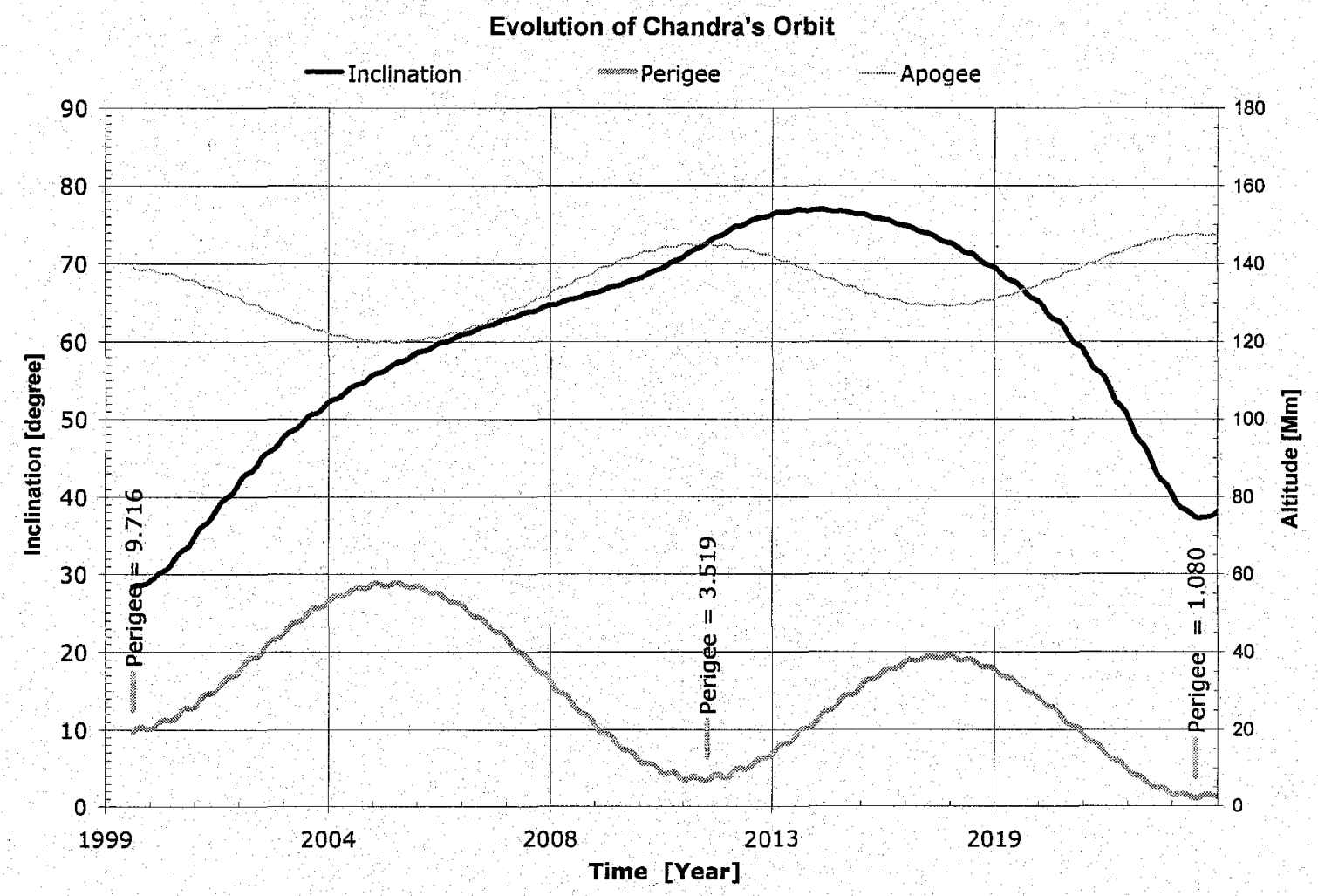

Figure 1: Evolution of Chandra's orbit and external exposure to trapped protons. The orbit's apogee, perigee, and inclination change substantially over the 25 years shown in the plot. 


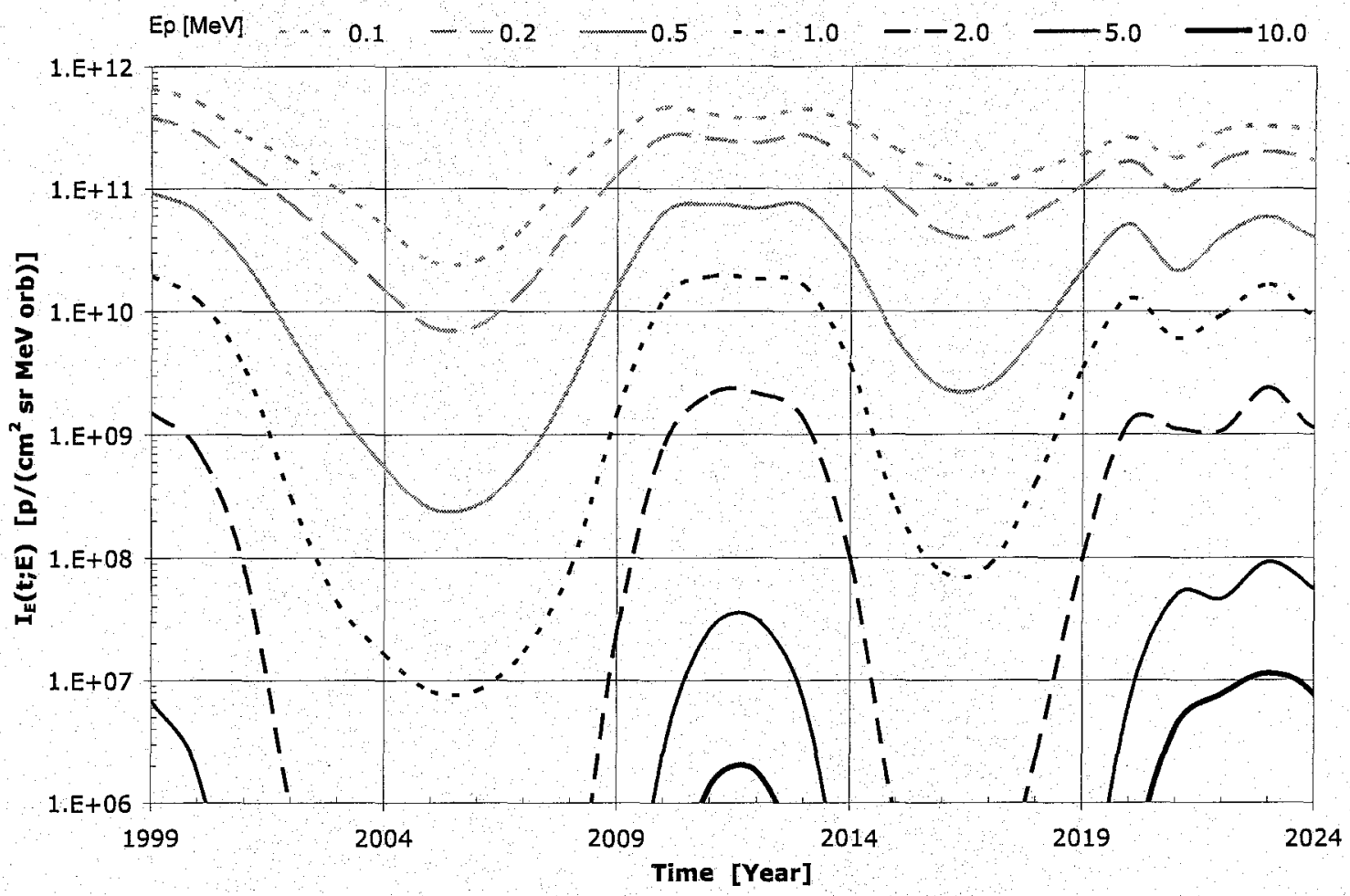

Figure 2: Evolution of external exposure to trapped protons in Chandra's orbit from 1999 August (first light) to 2024 August.

As Figure 1 illustrates, the perigee of Chandra's orbit varies about its mean value $(\approx 16 \mathrm{Mm})$ quasi-sinusoidally with approximately a 13-y period and 13-Mm amplitude. At 6 years into the mission, the perigee was near a maximum. Consequently, Chandra's exposure to the Earth's radiation belts was substantially less at that time than at the start of the mission; but it will now increase. Figure 2 shows the trapped-proton (external) orbital fluence, obtained using the SPace ENVironment Information System ${ }^{11}$ (SPENVIS) tool to propagate Chandra's orbit through the standard AP8 radiation environment. If ACIS were left in the focal position during perigee passes, the rate of radiation damage due to low-energy $(0.1-0.5 \mathrm{MeV})$ trapped protons varies by about 2 orders of magnitude. Of course, ACIS will never again be exposed to trapped protons during a perigee pass. Indeed, the command load always instructs the SIM translation table to remove the ACIS from focal position by about $70-\mathrm{Mm}$ altitude $\left(12 R_{\oplus}\right.$ geocentric). Thus, the low-energy (weakly penetrating) trapped protons that caused the initial damage to the front-illuminated CCDs will produce no additional CTI increase.

\subsection{Radiation-protection program}

The obvious radiation-protection strategy is to limit exposure of the ACIS to high proton-flux environments. In order to accomplish this, the radiation-protection program (Paper $\mathrm{I}^{10}$ ) employs scheduled $(\$ 2.2 .1)$, manual $(\$ 2.2 .2)$, and autonomous ( $\$ 2.2 .3)$ radiation safing. In each case, radiation-safing actions are the same - stop science observations, translate ACIS into next-in-line (NIL) position, power down ACIS video boards, and ramp down HRC high voltage.

\subsubsection{Scheduled protection}

Nominally once a week, the Chandra Operations Control Center (OCC) at the Chandra X-ray Center (CXC) transmits through NASA's Deep-Space Network (DSN) a command load for Chandra's on-board computer (OBC) to execute autonomously all science observations and other scheduled operations. Prior to radiation-zone ingress, each load explicitly safes the ACIS and HRC against radiation damage; after egress, the command load resumes science 
observations. In order to set the time to protect the science instruments and the time to resume science observations, the mission planners utilize the standard AP8/AE8 environment, with AE8 being the more restrictive. Because these models are not perfect and because the radiation zones-especially the outer electron zone-exhibit substantial transient and localized variations, it is necessary to pad the AE8-predicted times by a few hours. The science operations team completed a study and implemented changes to optimize these radiation-zone pad times.

Scheduled protection prevents the rapid increase in FI-CCD CTI suffered during proton-belt transits. However, transient events elsewhere in the radiation environment-solar radiation storms, solar-wind shocks, and geomagnetic storms - contain proton fluences that would noticeably degrade CCD performance over time. Thus, the Chandra team developed and implemented additional steps ( $\$ 2.2 .2$ and $\$ 2.2 .3)$ to limit exposure of the ACIS to such events.

\subsubsection{Manual intervention}

Chandra has an on-board radiation monitor that the $\mathrm{OBC}$ uses autonomously $(\$ 2.2 .3)$ to protect the science instruments against certain severe radiation events - such as inadvertent, unprotected radiation-belt entry. However, the radiationmonitor data (like all Chandra data) are available in real time or via data dump only during DSN contacts, typically for about an hour every 8 hours or so. Furthermore, the Chandra radiation monitor is not sensitive to the low-energy protons responsible for damaging the ACIS CCDs. For these reasons, the team implemented an extensive monitoring program using real-time environments data from other spacecraft, available through the National Oceanographic and Atmospheric Administration (NOAA) Space Environment Center (SEC).

The GOES proton monitor and the Advanced Composition Explorer ${ }^{12}$ (ACE) Solar Isotope Spectrometer ${ }^{13}$ (SIS) transmit real-time data for protons more energetic than $5 \mathrm{MeV}$ and $10 \mathrm{MeV}$, respectively. GOES satellites are in geostationary orbits, while $A C E$ is near the first Lagrange (L1) point. In order to get real-time data at altitudes more representative of Chandra's orbit, the science operations team has also arranged to retrieve near-real-time data from the radiation monitor aboard ESA's XMM-Newton. Because solar protons more energetic than about $5 \mathrm{MeV}$ typically penetrate the Earth's magnetosphere down to geosynchronous orbit, the GOES proton monitor serves as an effective real-time predictor between DSN contacts, of the count rate in the corresponding proton channels of Chandra's onboard radiation monitor $(\$ 2.2 .3)$.

The $A C E$ Electron, Proton, and Alpha Monitor ${ }^{14}$ (EPAM) provides the most relevant data-namely, the low-energy (0.05-2-MeV) proton spectrum in the solar wind. To supplement the $A C E$ EPAM data, MSFC's space environments group developed the Chandra Radiation Model $^{15}$ (CRM). Used in conjunction with $A C E$ data, the CRM permits realtime estimation of the $0.14-\mathrm{MeV}$-proton spectral intensity throughout Chandra's orbit, which passes through 3 spaceenvironment regions - (1) solar wind, (2) magnetosheath, and (3) magnetosphere. Recently, the space environments group updated ( $\$ 3.1)$ the CRM.

The Chandra team carefully monitors the $0.14-\mathrm{MeV}$-proton orbital fluence measured by the $A C E$ EPAM (in the solar wind) and estimated by the real-time CRM, and issues an alert (via pager and e-mail) if the 2-hour fluence exceeds a specified value. Based upon this or other automated alerts or upon concerns of personnel watching the radiation environment, the team convenes via teleconference, evaluates the radiation environment, and decides whether to intervene manually upon the next scheduled DSN contact, by activating the radiation-protection stored command sequence (SCS107). The team re-convenes as needed until the radiation threat has passed, the mission planners have built a new load, and science observations have resumed.

\subsubsection{Autonomous protection}

The Electron, Proton, Helium Instrument ${ }^{16}$ (EPHIN, \$3.2.1) -flight spare for the SOlar \& Heliospheric Observatory $(S O H O)$ - serves as Chandra's on-board radiation monitor. EPHN collects data in 4 electron channels $(0.25-10.4$ $\mathrm{MeV}$, 4 proton channels $(4.3-53 \mathrm{MeV}), 4$ helium channels $(4.3-53 \mathrm{MeV} / \mathrm{n})$, and an integral channel. During DSN contacts, Chandra downloads all EPHIN data for CXC use and for space-physics science by the EPHIN science team.

During normal Chandra operations, the process "RADMON" runs on the OBC, sampling and evaluating 3 EPHIN channels - P4 (4.3-7.8-MeV proton), P41 (41-53-MeV proton), and E1300 (2.6-6.2-MeV electron)-that serve as the real-time radiation monitor. If the count rate in any of these 3 channels exceeds its respective pre-set threshold for $\mathrm{N}$ consecutive 65.6-s samples, the $\mathrm{OBC}$ autonomously activates the radiation-protection stored command sequence 
(SCS107). Recently, the Chandra team increased the number $\mathrm{N}$ - from 3 to 10 -of over-threshold samples required to activate SCS107, in order to reduce spurious or otherwise unnecessary triggers.

Due to elevated EPHIN temperatures resulting from degradation of its multi-layer insulation (MLI), the data in some EPHIN channels may eventually become noisy. In order to prepare for this possibility, the CXC and HRC teams recently prepared contingency plans for using the $\mathrm{HRC}$ anti-coincidence shield as a radiation monitor (\$3.2).

\subsection{Radiation events}

During the first eight years of Chandra operations, there were 64 radiation protection (SCS 107) events. The team manually protected the science instruments 20 times for 1.911-Ms lost science; Chandra autonomously protected the science instruments 44 times for 5.240-Ms lost science. Most SCS107 triggers resulted from solar-energetic-particle (SEP) events accompanying major solar flares: This is the type of hard-proton event for which the radiation monitor was originally intended. Several SCS107 triggers occurred just prior to scheduled protection (\$2.2.1) for radiation-zone ingress: These events resulted from electron-flux spikes that present no danger to the science instruments. A few SCS107 triggers happened immediately following re-enabling of the RADMON process following a radiation safing: In these cases, a single above-threshold sample (either real or stale data) triggered SCS107 before the above-threshold counter had cleared from the previous radiation event.

Table 1: Radiation protection events

\begin{tabular}{|c|c|c|c|}
\hline SCS107 time & Re-start time & Time & \\
\hline $2000: 160: 09: 41$ & $2000: 161: 12: 30$ & 97.2 & $\mathrm{~A}$ \\
\hline $2000: 195: 11: 29$ & $2000: 196: 04: 47$ & 61.2 & manual \\
\hline $2000: 196: 22: 00$ & $2000: 198: 23: 15$ & 171.8 & manual \\
\hline $2000: 257: 19: 15$ & $2000: 258: 21: 00$ & 93.5 & $\mathrm{~A}$ \\
\hline $2000: 313: 23: 26$ & $2000: 316: 06: 50$ & 141.7 & $\mathrm{~A}$ \\
\hline $2000: 331: 19: 46$ & $2000: 333: 10: 00$ & 137.9 & $\mathrm{~A}$ \\
\hline $2001: 093: 02: 22$ & $2001: 096: 05: 17$ & 215.5 & $\mathrm{~A}$ \\
\hline $2001: 098: 04: 40$ & $2001: 099: 14: 28$ & 122.4 & manual \\
\hline $2001: 102: 03: 51$ & $2001: 103: 07: 16$ & 49.0 & $\mathrm{~A}$ \\
\hline $2001: 106: 00: 27$ & $2001: 107: 04: 00$ & 99.5 & $\mathrm{~A}$ \\
\hline $2001: 118: 04: 52$ & $2001: 119: 04: 40$ & 33.8 & manual \\
\hline $2001: 228: 02: 08$ & $2001: 229: 00: 30$ & 79.2 & $\mathrm{~A}$ \\
\hline $2001: 267: 14: 29$ & $2001: 273: 16: 50$ & 471.4 & $\mathrm{~A}$ \\
\hline $2001: 275: 06: 40$ & $2001: 276: 12: 00$ & 105.8 & $\mathrm{~A}$ \\
\hline $2001: 294: 15: 38$ & $2001: 296: 08: 00$ & 95.0 & manual \\
\hline $2001: 298: 23: 17$ & $2001: 300: 10: 40$ & 117.5 & manual \\
\hline $2001: 308: 17: 37$ & $2001: 312: 20: 07$ & 300.3 & $\mathrm{~A}$ \\
\hline $2001: 324: 03: 15$ & $2001: 325: 14: 33$ & 68.8 & manual \\
\hline $2001: 327: 01: 45$ & $2001: 329: 16: 30$ & 167.5 & $\mathrm{~A}$ \\
\hline $2001: 360: 06: 30$ & $2001: 362: 00: 00$ & $97: 4$ & $\mathrm{~A}$ \\
\hline $2002: 011: 03: 17$ & $2002: 011: 21: 15$ & 64.8 & manual \\
\hline $2002: 077: 13: 51$ & $2002: 079: 07: 34$ & 116.7 & manual \\
\hline $2002: 107: 12: 55$ & $2002: 108: 05: 30$ & 42.1 & manual \\
\hline $2002: 109: 14: 15$ & $2002: 110: 17: 43$ & 83.5 & manual \\
\hline $2002: 111: 04: 52$ & $2002: 113: 21: 30$ & 171.8 & $\mathrm{~A}$ \\
\hline $2002: 143: 09: 58$ & $2002: 144: 11: 45$ & 93.6 & $\mathrm{~A}$ \\
\hline $2002: 198: 12: 32$ & $2002: 199: 15: 45$ & 95.2 & $\mathrm{~A}$ \\
\hline $2002: 230: 18: 43$ & $2002: 231: 19: 48$ & $90: 0$ & manual \\
\hline $2002: 236: 02: 11$ & $2002: 237: 13: 26$ & 126.0 & $\mathrm{~A}$ \\
\hline $2002: 314: 03: 20$ & $2002: 315: 09: 54$ & 55.4 & $\mathrm{~A}$ \\
\hline $2002: 331: 00: 02$ & $2002: 332: 03: 30$ & 97.2 & manual \\
\hline $2003: 107: 20: 36$ & $2003: 108: 11: 40$ & 5.7 & $\mathrm{~A}$ \\
\hline & & & \\
\hline
\end{tabular}

\begin{tabular}{|c|c|c|c|}
\hline SCS107 time & $\begin{array}{c}\text { Re-start } \\
\text { time }\end{array}$ & Time & \\
\hline $2003: 120: 23: 37$ & & 23.3 & $\mathrm{~A}$ \\
\hline $2003: 128: 20: 08$ & & 50.8 & $\mathrm{~A}$ \\
\hline $2003: 149: 21: 47$ & & 74.1 & manual \\
\hline $2003: 173: 18: 48$ & & 10.8 & $\mathrm{~A}$ \\
\hline $2003: 213: 11: 39$ & & 7.8 & $\mathrm{~A}$ \\
\hline $2003: 297: 13: 34$ & & 61.1 & manual \\
\hline $2003: 299: 19: 22$ & & 90.4 & $\mathrm{~A}$ \\
\hline $2003: 301: 13: 02$ & & 172.3 & $\mathrm{~A}$ \\
\hline $2003: 306: 20: 38$ & & 293.2 & $\mathrm{~A}$ \\
\hline $2003: 326: 01: 24$ & & $112: 6$ & manual \\
\hline $2003: 336: 17: 31$ & & 139.8 & $\mathrm{~A}$ \\
\hline $2004: 009: 13: 45$ & & 68.2 & manual \\
\hline $2004: 021: 11: 51$ & & 118.8 & manual \\
\hline $2004: 181: 19: 05$ & & 0.8 & $\mathrm{~A}$ \\
\hline $2004: 208: 03: 54$ & & 3.6 & $\mathrm{~A}$ \\
\hline $2004: 208: 21: 59$ & & 82.8 & $\mathrm{~A}$ \\
\hline $2004: 210: 19: 24$ & & 5.3 & $\mathrm{~A}$ \\
\hline $2004: 213: 12: 02$ & & 3.3 & $\mathrm{~A}$ \\
\hline $2004: 257: 21: 38$ & & 92.9 & $\mathrm{~A}$ \\
\hline $2004: 312: 20: 14$ & & 183.2 & manual \\
\hline $2004: 315: 16: 41$ & & 104.7 & $\mathrm{~A}$ \\
\hline $2005: 016: 14: 44$ & & 381.7 & $\mathrm{~A}$ \\
\hline $2005: 096: 00: 59$ & & 6.0 & $\mathrm{~A}$ \\
\hline $2005: 134: 24: 19$ & & 66.9 & $\mathrm{~A}$ \\
\hline $2005: 213: 13: 16$ & & 128.1 & manual \\
\hline $2005: 234: 19: 40$ & & $283: 1$ & $\mathrm{~A}$ \\
\hline $2005: 251: 01: 57$ & & 286.5 & $\mathrm{~A}$ \\
\hline $2005: 257: 01: 06$ & & 95.2 & $\mathrm{~A}$ \\
\hline $2006: 079: 01: 32$ & & 10.3 & $\mathrm{~A}$ \\
\hline $2006: 340: 16: 16$ & & 225.0 & $\mathrm{~A}$ \\
\hline $2006: 344: 08: 59$ & & 84.0 & $\mathrm{~A}$ \\
\hline $2006: 347: 22: 44$ & & 115.6 & $\mathrm{~A}$ \\
\hline & & & \\
\hline
\end{tabular}




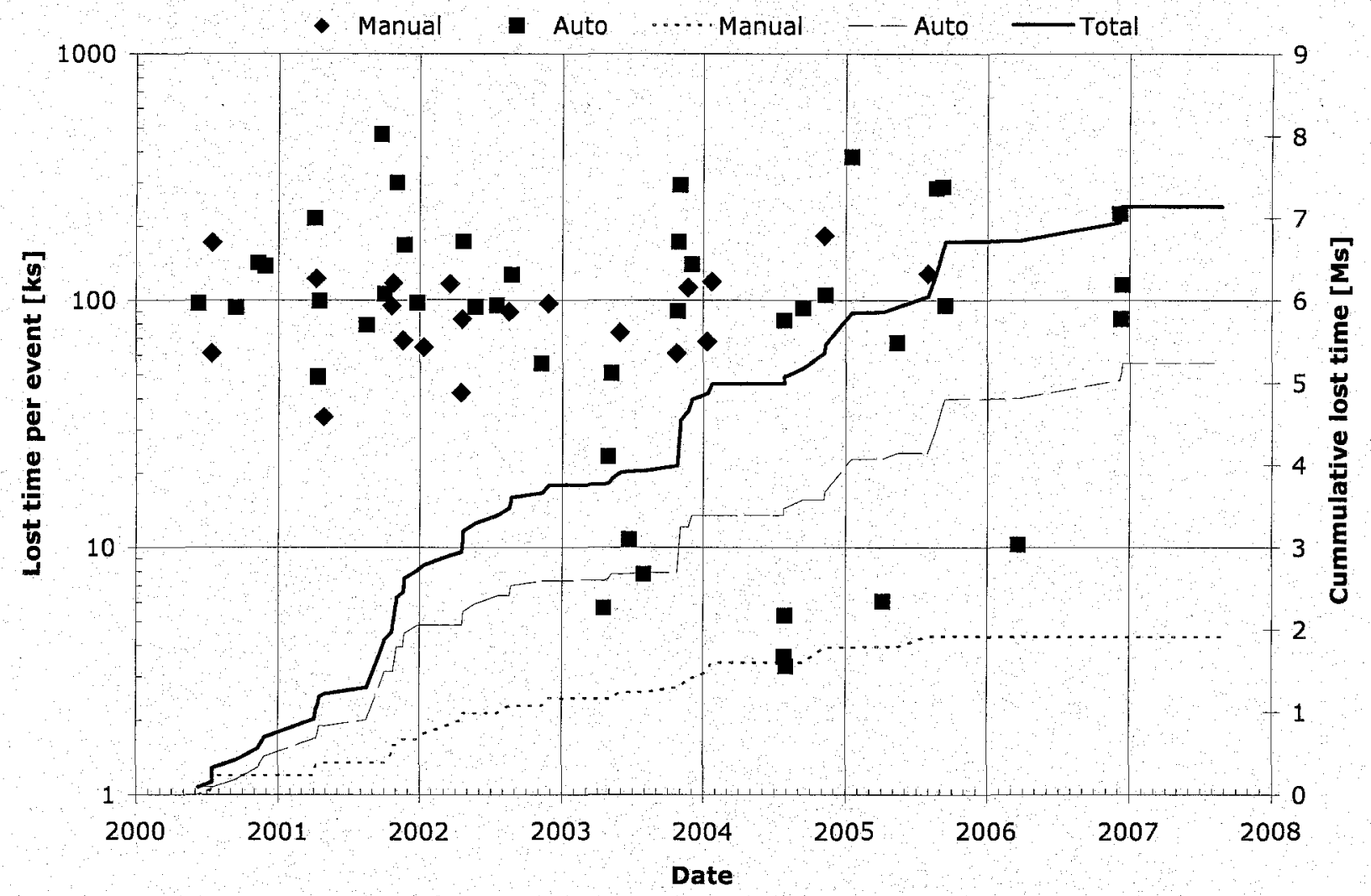

Figure 3: Science time lost to protecting against radiation events during first 8 years of Chandra operations.

\subsection{Charge-transfer inefficiency}

The radiation-protection program $(\$ 2.2)$ has successfully reduced the rate of CTI increase to manageable levels. Figure 4 displays the CTI since 2000 January, after cooling the ACIS focal plane to its current operating temperature $\left(-120^{\circ} \mathrm{C}\right)$. Displayed CTI measurements ${ }^{17}$ are from the ACIS ( $\mathrm{Fe}^{55}$ ) external calibration source (ECS), which ACIS views while stowed in the NIL position, and are corrected for sacrificial charge ${ }^{18}$ from cosmic rays. Currently, the FI-CCD CTI $=$ $16.0 \times 10^{-5}$; the BI-CCD CTI $=2.0 \times 10^{-5}$. Since 2001 January, the CTI has increased at $0.29 \times 10^{-5} / \mathrm{y}(1.8 \% / \mathrm{y})$ for FI CCDs and at $0.08 \times 10^{-5} / \mathrm{y}(4.2 \% / \mathrm{y})$ for BI CCDs - slightly less than the average rates since 2000 January. Hence, the rate of CTI increase is sufficiently small to allow ACIS to continue to provide spectrometric imaging for decades. 

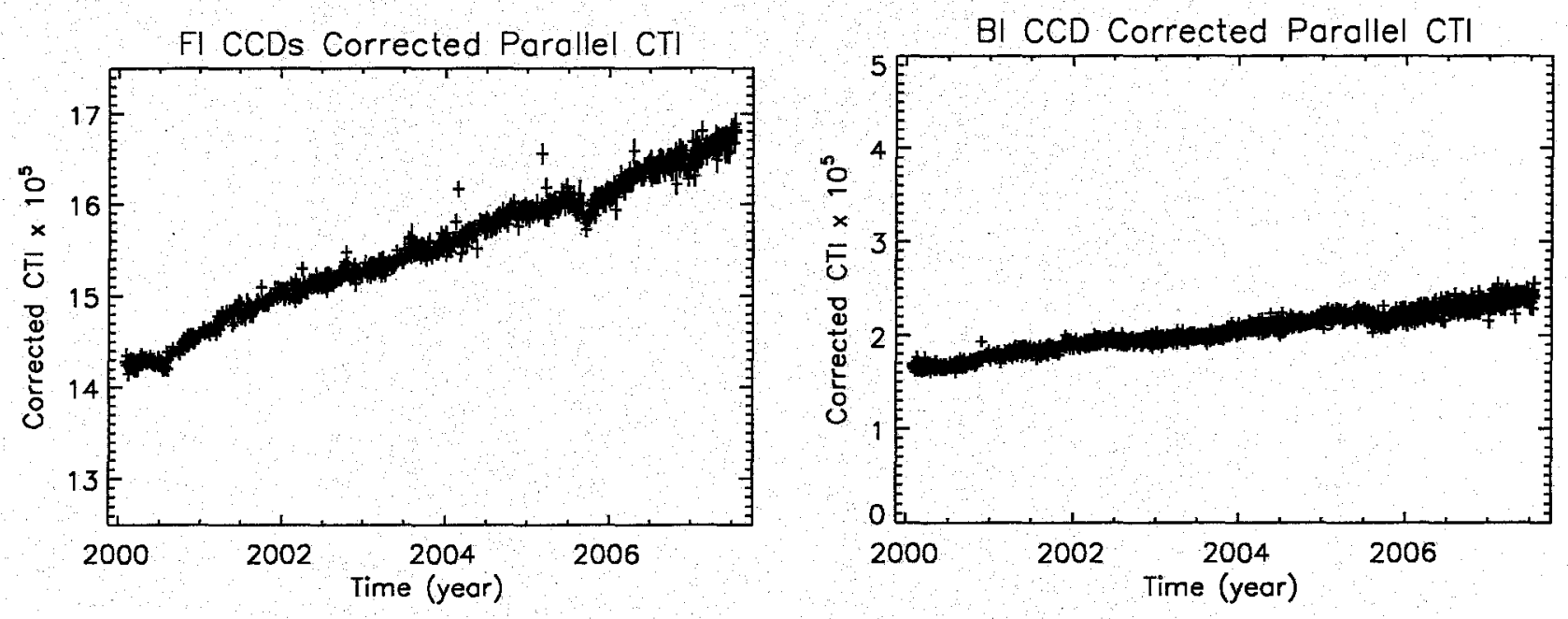

Figure 4: Charge transfer inefficiency (CTI) of ACIS CCDs at $5.9 \mathrm{keV}(\mathrm{Mn}-\mathrm{K} \alpha)$, for the focal plane at $-120^{\circ} \mathrm{C}$, corrected for cosmic-ray sacrificial charge. Left panel shows the average CTI of the 4 front-illuminated CCDs comprising the imaging (I) array; right panel, the CTI of the back-illuminated CCD (S3) at the center of the spectroscopy (S) array.

CXC data processing now implements a CTI corrector, originally developed by the ACIS team. ${ }^{19,20}$ The CTI corrector removes the non-stochastic part of the CTI-induced position-dependent gain error. Thus, the FI CCDs continue to provide high-resolution imaging spectrometry ${ }^{21}$-albeit with poorer energy resolution than originally expected. The CTI of the BI devices is an order of magnitude less than that of the radiation-damaged FI CCDs, but currently increasing at about 0.3 the rate of that of the FI CCDs. In contrast, the unprotected radiation-belt passes, which produced the initial damage in the FI devices, resulted in no measurable increase in the CTI of the BI CCDs. This indicates ${ }^{17}$ that the average spectrum of protons reaching the ACIS CCDs is now significantly harder than during the unprotected radiation-belt passes. Note that $1.9 \times 10^{-4} \mathrm{~g} \mathrm{~cm}^{-2}$ shields the FI charge transfer channel from protons under $0.1 \mathrm{MeV} ; 1.2 \times 10^{-2} \mathrm{~g} \mathrm{~cm}^{-2}(45-\mu \mathrm{m}$ silicon) shields the BI charge transfer channel from protons under $2.2 \mathrm{MeV}$.

\section{ENHANCEMENTS}

We introduced several enhancements to the radiation-protection program (\$2.2) since Paper I $^{10}$ For scheduled protection (\$2.2.1), we reduced pad times on AE\& predictions of radiation-zone ingress and egress so as to increase science time. For manual intervention (\$2.2.1), we added access to real-time data from the XMM-Newton radiation monitor; we updated the Chandra Radiation Model (CRM) to include data through 2004 that more fully populates relevant parameter space ( $\$ 3.1)$. For autonomous protection $(\$ 2.2 .3)$, we increased the number of EPHIN samples required to activate radiation safing, in order to reduce unnecessary triggers; we developed contingency procedures to use the HRC anti-coincidence shield as a radiation monitor if EPHIN data become problematic $(\$ 3.2)$.

\subsection{Chandra radiation model}

The Chandra Radiation Model ${ }^{15}$ (CRM) is a 3-dimensional map of the $0.14-\mathrm{MeV}$-proton spectral intensity (Figure 5). We derive the CRM from a correlation of archival data-from the Geotail Energetic Proton and Ion Composition (EPIC) detector ${ }^{22}$ and from the Polar Comprehensive Energetic-Particle Pitch Angle Distribution (CEPPAD) instrument ${ }^{23}$ - with the geomagnetic planetary index $\mathrm{Kp}$. In order to populate fully the data cube throughout the domain of geocentric radii 8-32 $R_{\oplus}$, we also map the particle distribution along the magnetic field - "field-line mapping". Recently, we updated the CRM to include Geotail EPIC and Polar CEPPAD data through 2004, which significantly increased data coverage for high-Kp periods. Figure 5 exhibits an equatorial slice in Geocentric Solar Magnetospheric (GSM) coordinates, of the updated CRM data cube for geomagnetic conditions characterized by $\mathrm{Kp}=1$ (quiet), 3 (moderate), 5 (minor storm), and 7 (strong storm). Note the three distinct radiation regions-(1) solar wind, (2) magnetopause, and (3) magnetosphere - separated by the bow shock and by the magnetopause, respectively. 

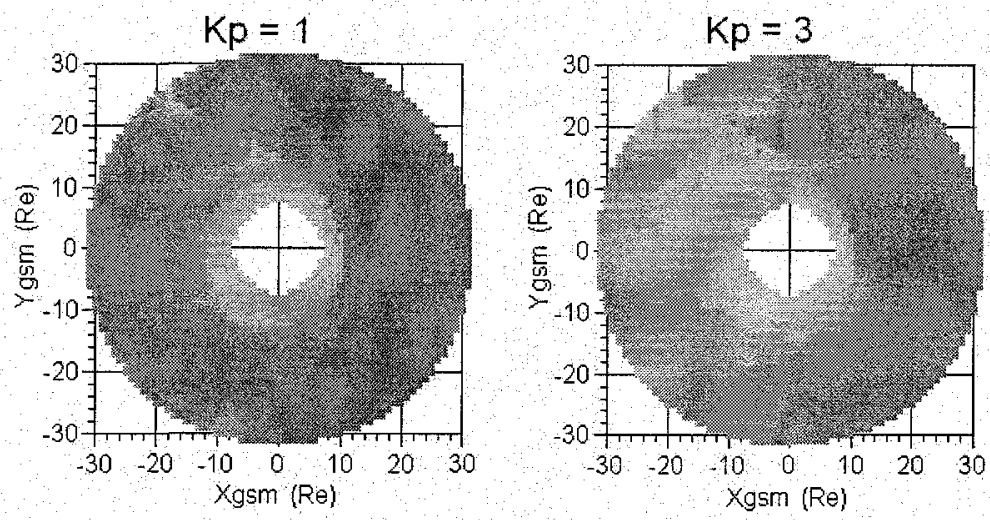

HCm?2-sec-sr-MeV
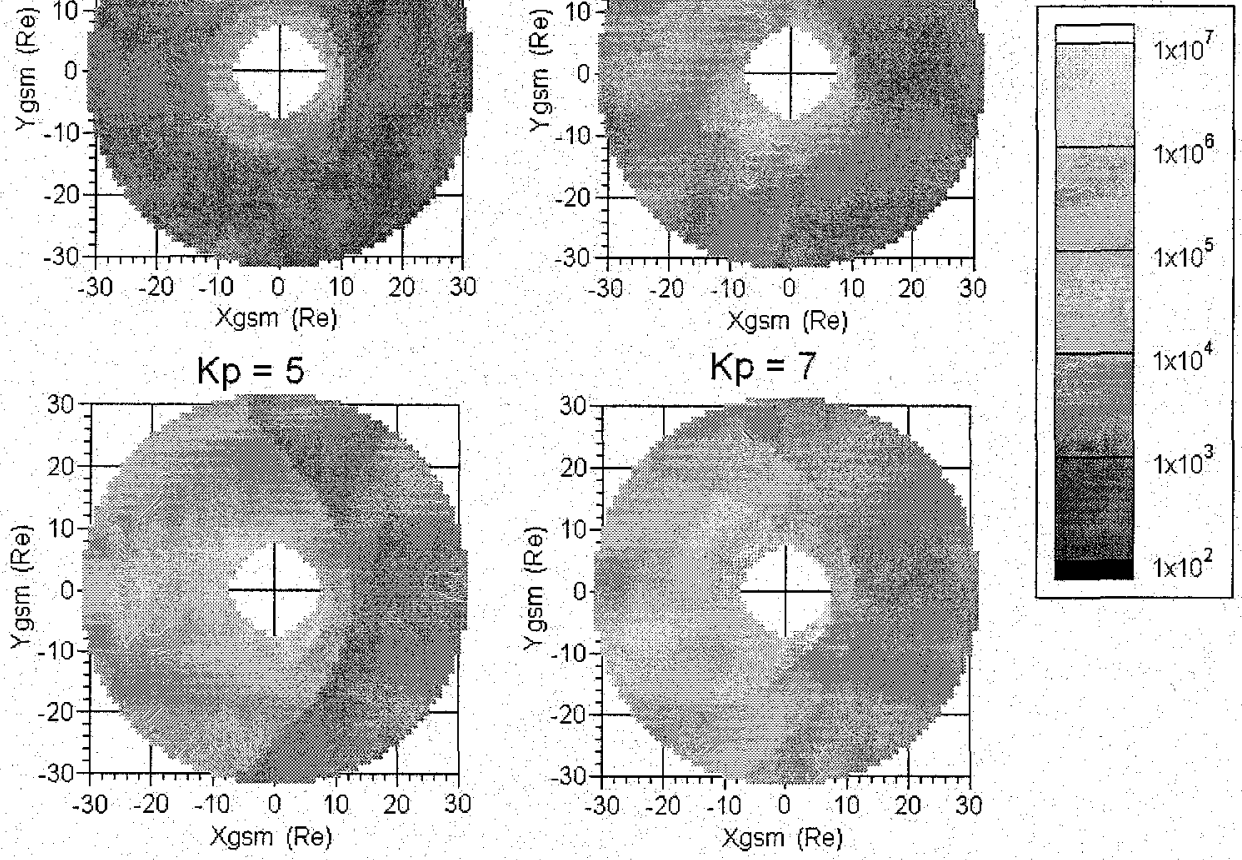

Figure 5. Chandra Radiation Model (CRM) for $0.14-\mathrm{MeV}$ protons at geocentric distances $8-32 \mathrm{R}_{\oplus}$ for 4 values of the geomagnetic planetary index $\mathrm{Kp}$. Each displayed map is an equatorial slice in geocentric solar magnetospheric (GSM) coordinates, with the sun on the $+X_{g s m}$ axis and the Earth's magnetic poles in the $X_{g s m}-Z_{g s m}$ plane.

We employ the CRM for multiple purposes. Mission planners now schedule radiation protection for perigee passes using radiation-zone pad times derived from a combined analysis of archival EPHIN and mean-CRM data. For realtime estimation, $A C E$ Solar-Wind Electron Proton Alpha Monitor ${ }^{24}$ (SWEPAM) data ${ }^{25}$ drives the SEC-provided (neuralnet) Costello $\mathrm{Kp}$ estimator ${ }^{26}$, which in turn drives our $\mathrm{Kp}$-dependent $\mathrm{CRM}$ estimator of proton intensity. This allows a real-time estimate of $0.14-\mathrm{MeV}$-proton fluence from the start of an orbit. For an assumed value of $\mathrm{Kp}$, we can also project the fluence for the rest of that orbit. If the estimated fluence exceeds a budgeted amount, we can decide whether to intervene ( $\$ 2.2 .2)$ by manually activating radiation safing (SCS107) at the next DSN contact.

Naturally, the $A C E$ EPAM $\mathrm{P} 3$ channel furnishes the best real-time estimator for the 0.14-MeV-proton spectral intensity when Chandra is in the solar wind. In the magnetosheath (between bow shock and magnetopause), the population may have contributions from shocked solar-wind protons and from protons leaking from the magnetosphere. In the magnetosphere, the population may have contributions from quasi-trapped magnetospheric protons and from solar-wind protons penetrating the magnetopause. Thus, we adopt a conservative approach and utilize a hybrid of real-time EPAM data and the real-time SWEPAM-Kp driven CRM estimate:

1. Solar wind $F_{1}(t)=F_{E P A M}(t)$

2. Magnetosheath $F_{2}(t)=2 \times F_{\text {EPAM }}(t)+F_{C R M}(K p(t))$

3. Magnetosphere $F_{3}(t)=F_{C R M}(K p(t))+1 / 2 \times F_{\text {EPAM }}(t)$

\subsection{EPHIN thermal degradation}

During the mission, the thermal-control properties of Chandra's silverized-Teflon multilayer insulation (MLI) have degraded, probably due to radiation damage during perigee passes and thermo-mechanical stresses during sun-angle changes. While thermal control of the Observatory itself has adequate margin, that of the Electron, Proton, Helium Instrument (EPHIN) does not. Designed to monitor solar energetic particles, EPHIN resides on the sunward side of the 
spacecraft, unshaded during normal operations. Temperatures experienced by EPHIN detectors and electronics will continue to rise as the MLI's solar absorptance further increases. Limiting sun angles to minimize near-normal illumination of EPHIN helps; however, this practice constrains mission planning and will eventually be insufficient. Thus, we expect that progressively higher temperatures will eventually degrade EPHIN's performance and efficacy as a radiation monitor. Here we consider $(\S 3.2 .1)$ the anticipated impact of rising EPHIN temperatures, describe $(\S 3.2 .2)$ potential use of the HRC anti-coincidence shield as a radiation monitor, compare (\$3.2.3) HRC anti-co rates and EPHIN rates; and discuss (\$3.2.4) contingency plans to replace EPHIN data used by RADMON (\$2.2.3) with HRC anti-co data.

The EPHIN is a critical component of the on-board radiation monitoring system. Continuing degradation of its thermalcontrol surfaces has lead to higher temperatures at a given attitude and shorter durations allowed at sun-facing attitudes when attempting to limit the maximum temperature. The elevated temperature is a concern due to the increased leakage currents at high temperature increasing the detector backgrounds. However, a more significant affect of the elevated temperatures is that the increased detector leakage currents result in a larger than planned for power-draw on the $+27 \mathrm{~V}$ rail power supply used to drive the detector high-voltage power supplies. This high power-draw causes the $+27 \mathrm{~V}$-rail supply to enter a current-limited state, with a reduced output voltage. The reduced $+27 \mathrm{~V}$-rail supply output voltage results in reduced output in the detector HV supplies. This anomalous behavior results in changes in EPHIN performance.

The EPHIN $+27 V$-rail supply enters the current-limited state when the detector temperatures reach $\sim 37 \mathrm{degC}$. The entrance is characterized by a sudden drop in the $+27 \mathrm{~V}$ output to typically less than half its nominal value. The $+27 \mathrm{~V}$ output drop is accompanied by a drop in the output of the HV supply for detector G (Figure 6, left panel), the PMT used to readout the guard counter. There is no telemetry for the output levels of the other HV power supplies but the presumably drop as well. The observed net effect of the HV reduction is a decrease in the detector G rate (Figure 6 right or Figure 7 left) and an increase in the rates of the four EPHIN electron channels (Figure 7 right) and the integral channel. These increases are due to the loss of the anti-coincidence signals from detector $\mathrm{G}$. The rates in the other EPHIN detectors do not currently appear to be affected by the (unmeasured) drop in their HV supplies. The output levels of the HV supplies for the other detectors do not appear to drop enough to impact their operation. As long as the $\mathrm{HV}$ output is sufficiently above the level required for full-depletion of the $\mathrm{Si}(\mathrm{Li})$ detectors should not decompensate. There is hysteresis in the recovery from the anomalous state and temperatures must fall below $\sim 29$ degC for the $+27 \mathrm{~V}$ rail supply to return to normal.

This anomalous EPHIN behavior initially lead us to schedule observations such that the maximum predicted EPHIN temperature would not trigger an entrance into the anomalous state. Unfortunately, as the thermal-control surfaces continued to degrade and a thermal constraint imposed by the spacecraft propulsion system removed the best attitude for cooling EPHIN from use, scheduled science-observing efficiency was noticeably reduced. Since the detector rates (other than that of detector $\mathrm{G}$ ) did not appear to be affected by the reduction of the HV and an analysis of the median detector pulse-heights for minimum-ionizing particles showed no significant degradation with reduced voltage it was decided in December 2005 to allow the EPHIN to routinely operate in the anomalous state. In order to reduce the possibility of unnecessary triggers of on-board radiation safing sequence due to false high-E1300 fluxes during times of anomalous performance, the E1300 threshold within RADMON had to be raised from $10 \mathrm{cts} / \mathrm{cm}^{\wedge} 2-\mathrm{s}-\mathrm{sr}$ to $20 \mathrm{cts} / \mathrm{cm}^{\wedge} 2$ s-sr. The EPHIN detector rates and pulse-heights are monitored for evidence of the onset of degradation.

One factor the aided the decision to permit the routine operation of the EPHIN in the anomalous state was the fact the the HRC was available as a replacement for on-board radiation monitoring. As described in our earlier paper[reference to 2005 paper], the HRC anti-coincidence shield rate can be used within the on-board computer's RADMON process to flag times of high fluxes of particle radiation. Figure 8 compares the $\mathrm{HRC}$ anti-coincidence shield rate to the E1300 and EPHIN P41 fluxes. As discussed in our earlier paper, we currently do not choose to activate HRC anti-coincidence monitoring within the RADMON process due to the possibility of an unnecessary trigger given the observed performance within the available dynamic range. 

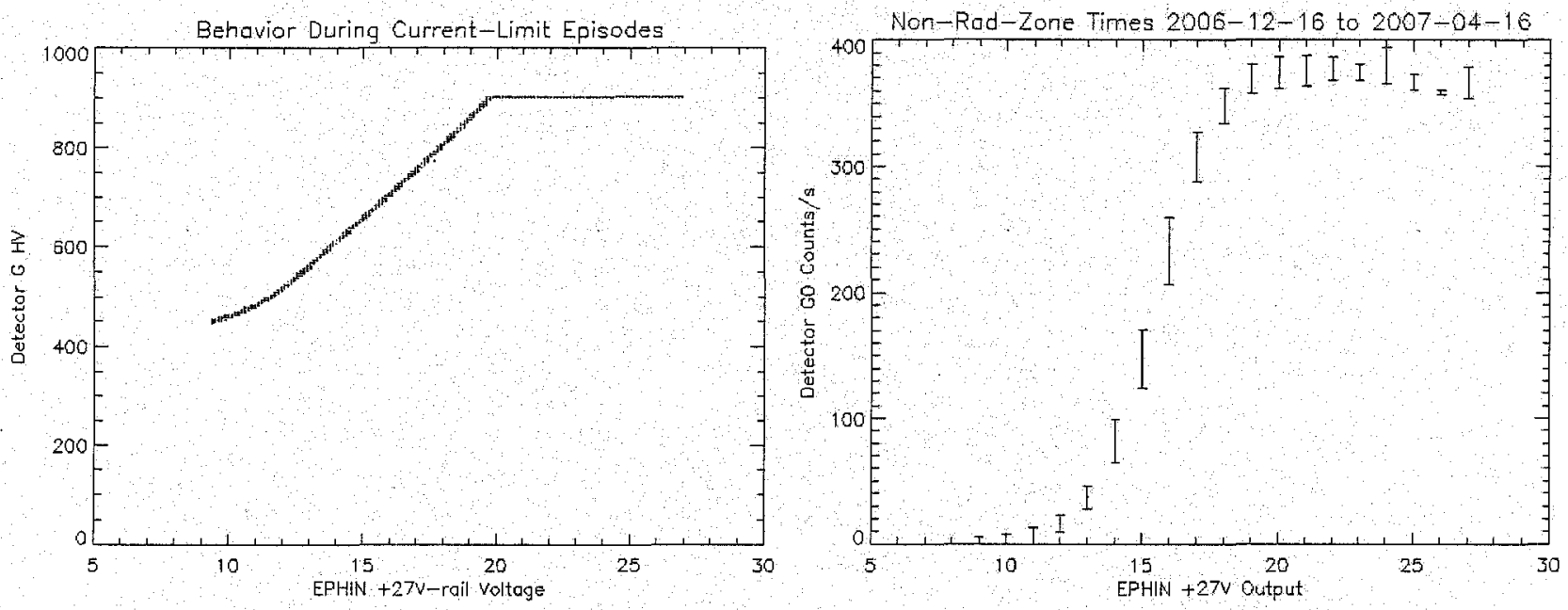

Figure 6: Consequences of rail-voltage drops due to high operating temperatures upon operation of the EPHIN guard (G) detector. The left panel shows the output of the detector $\mathrm{G}$ high-voltage power supply as a function of the input $+27 \mathrm{~V}$ level during times when the EPHIN $+27 \mathrm{~V}$ was operating in an anomalous state. The right panel shows the observed detector $\mathrm{G}$ rate as a function of $+27 \mathrm{~V}$-rail supply output during anomalous operations. Times when Chandra was within the Earth's radiation belts have been excluded.

\subsubsection{Potential impacts}

The EPHIN instrument ${ }^{16}$ uses coincidences in a stack of 6 detectors -3 ion-implanted Si detectors (" $A$ ", " $B$ ", and " $F$ ") and 3 lithium-drifted $\mathrm{Si}[\mathrm{Si}(\mathrm{Li})]$ detectors ("C", "D", and "E") plus a guard detector ("G", a scintillator read by a photomultiplier tube, PMT), to assign the species and energy of incident particles. The EPHIN Input/Output (EIO) interfaces between EPHIN and the spacecraft and collects EPHIN data-including counts in a number of coincidence channels corresponding to various particle energy ranges - for inclusion in telemetry. Currently, the OBC RADMON process watches 3 of 13 EPHIN coincidence channels-E1300, P4, and P41 (\$2.2.3).

The EPHIN team anticipates that elevated temperatures will eventually degrade performance of the $\mathrm{Si}(\mathrm{Li})$ detectors through more rapid Li diffusion. Further, elevated temperatures have raised the detectors' leakage currents, triggering episodes of reduced high voltage: This also would increase the Li diffusion rate if the bias drops too low. The expected degradation symptom is increased noise in the $\mathrm{Si}(\mathrm{Li})$ detectors. This may ultimately render detectors $\mathrm{C}, \mathrm{D}$, and $\mathrm{E}$ unusable, thus making EPHIN channels using those detectors - i.e., the higher energy channels - problematic. However, there are steps to mitigate the adverse impact of increased $\mathrm{Si}(\mathrm{Li})$-detector noise upon the radiation-protection program e.g., adjusting RADMON trigger levels, averaging samples, or watching alternate EPHIN channels. 

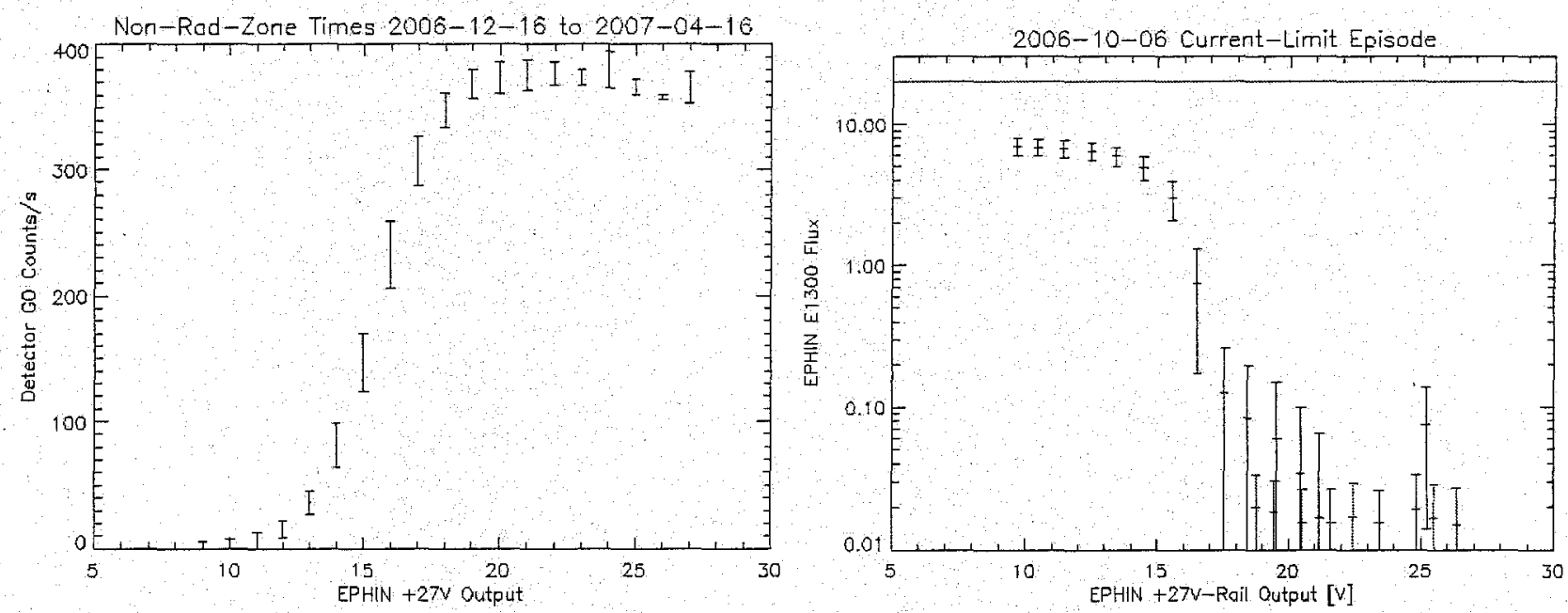

Figure 7: Consequences of rail-voltage drops and resulting (temporary) anomalous behavior of the EPHIN guard (G) detector. The left panel shows the observed detector $\mathrm{G}$ rate as a function of $+27 \mathrm{~V}$-rail supply output during anomalous operations. Times when Chandra was within the Earth's radiation belts have been excluded. The right panel displays the eported E1300 flux as a function of $+27 \mathrm{~V}$-rail supply output during anomalous operation. The horizontal line at $20 \mathrm{cts} / \mathrm{s}$ is the current threshold for E1300 triggers of SCS107.

\subsubsection{HRC anti-coincidence shield}

The HRC anti-coincidence ("anti-co") shield comprises plastic scintillator blocks that surround the detector housing on 5 sides, read by 1 of 2 photomultiplier tubes (PMTs). The purpose of the anti-co shield is to detect charged particles that penetrate the microchannel-plate (MCP) detectors, in order to distinguish (through coincidence) charged-particle events in the MCP from x-ray events and then to veto those events in the HRC electronics. Although the HRC team planned using the HRC anti-co shield and MCP detector total event rates as radiation-monitor inputs, implementation halted during pre-launch software development after incorporation of EPHIN as Chandra's radiation monitor. By design, EPHIN is a more versatile particle counter than the HRC anti-co shield and it has a much larger dynamic range.

HRC A-side and B-side electronics each send anti-co rates and total MCP rates to the OBC: Only data from the active side (now A) are meaningful. Recently, the flight operations team patched flight software to substitute these 4 HRC channels for the 4 EPHIN helium channels in the telemetry. If needed, the flight operations team will patch RADMON to substitute an HRC channel for one of the 3 watched EPHIN channels. Complete failure of EPHIN would require an additional patch so that the RADMON process would ignore the EPHIN hardware failure: Currently, RADMON will activate SCS107 if the OBC does not receive an "alive" signal from EPHIN through the EIO and from the EIO.

\subsubsection{EPHIN-HRC-anti-co comparison}

In order to determine optimal use of the HRC anti-co shield as a radiation monitor, the science operations and HRC teams conducted a comparative study of available EPHIN and HRC-anti-co data. Although the EPHIN operates throughout each orbit, the HRC high voltage (HV) is always "off" during radiation-zone transit. During the first year of the mission, the HRC HV remained "on" outside the radiation zone. Subsequently, the HRC HV was "on" only in conjunction with HRC observations, so as to maximize the operating life of the PMT. As concern over potential EPHIN degradation grew, we resumed collecting HRC anti-co data at all times outside the radiation zone, but at various reduced HV levels when the HRC was not engaged in a science observation.

Using available data, we compared HRC anti-co rates with rates in the 3 EPHIN channels-P4 (4.3-7.8-MeV proton), P41 (41-53-MeV proton), and E1300 (2.6-6.2-MeV electron) - watched by the RADMON process ( $\$ 2.2 .3)$. Figure 8 exhibits the scatter plots of raw (OBC ingested) HRC anti-co rates versus $\mathrm{P} 4$ spectral intensity (left) and versus P41 spectral intensity (right). The vertical line in each plot demarcates the respective threshold for RADMON to activate SCS107. For all anti-co data in Figure 8, the PMT HV had been set to step 8, its nominal setting for operations. Data 
collected at lower PMT HV steps exhibits the same behavior as shown in these plots. Not surprisingly, the HRC anti-co data correlates best with data from EPHIN's highest energy proton channel (P41)-i.e., the most penetrating radiation. The correlation with other EPHIN channels becomes progressively worse for less penetrating radiation.
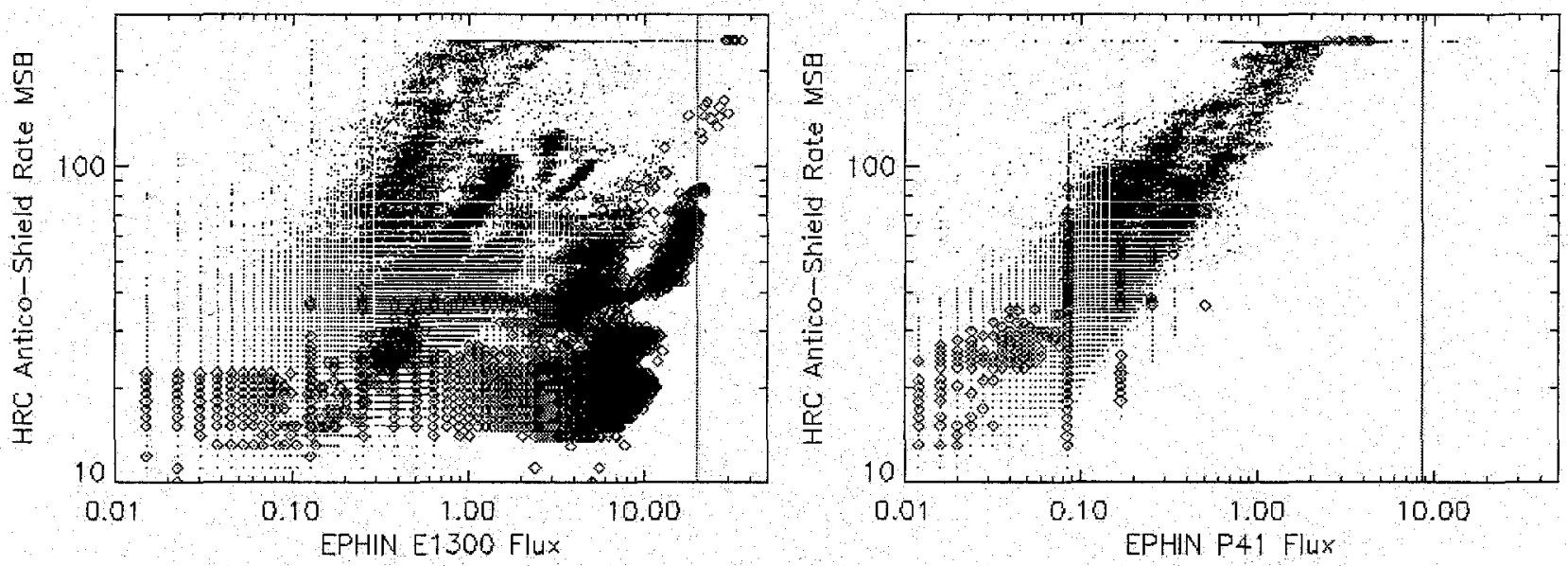

Figure 8: HRC anti-coincidence shield rate versus EPHIN E1300 flux (left) and P41 flux (right). Diamonds denote data acquired during anomalous operation of the EPHIN $+27 \mathrm{~V}$-rail supply. The vertical lines indicate the respective RADMON thresholds for autonomous activation of radiation protection (SCS107). HRC anti-co rates are in analogdigital units (ADU); EPHIN, \#/( $\mathrm{cm}^{2} \mathrm{~s}$ sr). Note the data-handling ceiling in anti-co rates at $248 \mathrm{ADU}$.

A potentially important limitation in the HRC anti-co data is a data-handling ceiling at 248 ADU (analog-digital units), set to avoid overflow of the single byte encoding the anti-co rate. Figure 8 clearly evidences this ceiling and that the anti-co rate typically reaches it before any EPHIN channel reaches its respective SCS107 threshold. The RADMON threshold for the HRC anti-co rate-necessarily less than its ceiling-might result in many unnecessary SCS107. activations. However, an investigation of EPHIN-triggered SCS107 and instances for which the anti-co rate would have triggered SCS107 alleviates this concern: Requiring 10 consecutive EPHIN samples above threshold, the anti-co rate would have triggered SCS107 for 6 of 14 EPHIN triggers, but would never have triggered without an EPHIN trigger.

\subsubsection{Contingency plans}

For Chandra radiation protection, HRC anti-co data are a poor substitute for EPHIN data. Whereas weakly penetrating radiation (0.1-0.5-MeV protons) causes most of the damage to the FI CCDs, the anti-co shield is sensitive only to penetrating radiation. Although not ideal, the EPHIN P4 channel (4.3-7.8-MeV proton) is a much better proxy for the lower energy protons of most concern. Further, while the HRC anti-co shield is a reasonably good proxy for the EPHIN P41 channel (\$3.2.3), P41 seldom triggers SCS107. Hence, the data-handling ceiling on HRC anti-co rates would probably not result in frequent unnecessary radiation safings. Indeed, the investigation of past SCS107 triggers found that the HRC anti-co rate would not have triggered about half the radiation safings triggered by EPHIN rates. On the other hand, the HRC anti-co rate would have triggered the other half and certainly would preclude an unprotected perigee pass - by far the most damaging radiation event for the ACIS FI CCDs.

Our strategy then is to employ HRC anti-co data in autonomous radiation protection only when they contribute capability that is no longer available from the EPHIN. Of course, a catastrophic failure of EPHIN will require that we use the HRC anti-co shield as the Chandra radiation monitor. However, the expected scenario (\$3.2.1) is a "graceful degradation" with the higher energy EPHIN channels - those relying most on the lithium-drifted silicon detectors in the detector stack - becoming noisy first. If fewer than 3 EPHIN channels are useful, RADMON will then watch HRC anti-co rates and the remaining EPHIN channel(s) for autonomous radiation safing. It is some comfort that the EPHIN $\mathrm{P} 4$ channel - the most relevant to radiation protection of the ACIS - is the least sensitive to thermal degradation.

\section{REFERENCES}


1 M.C. Weisskopf, T.L. Aldcroft, M. Bautz, R.A. Cameron, D. Dewey, J.J Drake, C.E Grant, H.L. Marshall, \& S.S. Murray, "An Overview of the Performance of the Chandra X-ray Observatory", Exp. Astron., 16, 1-68, 2003.

M.C. Weisskopf, B. Brinkman, C. Canizares, G. Garmire, S. Murray, \& L.P. Van Speybroeck, "An Overview of the Performance and Scientific Results from the Chandra X-Ray Observatory", Pub. Astron. Soc. Pacific, 114, 1-24, 2002.

3. M.C. Weisskopf, H.D. Tananbaum, L.P. Van Speybroeck, \& S.L. O'Dell, "Chandra X-ray Observatory (CXO): overview", Proc. SPIE, 4012, 2-16, 2000.

S.S. Murray, G.K. Austin, J.H. Chappell, J.J. Gomes, A.T. Kenter, R.P. Kraft, G.R. Meehan, M.V. Zombeck, G.W. Fraser, \& S. Serio, "In-flight performance of the Chandra High-Resolution Camera", Proc. SPIE, 4012, 68-80, 2000 .

G.P. Garmire, M.W. Bautz, P.G. Ford, J.A. Nousek, \& G.R. Ricker Jr., "Advanced CCD Imaging Spectrometer (ACIS) instrument on the Chandra X-ray Observatory", Proc. SPIE, 4851, 28-44, 2003.

G.Y. Prigozhin, S.E. Kissel, M.W. Bautz, C.E. Grant, B. LaMarr, R.F. Foster, \& G.R. Ricker, "Radiation damage in the Chandra X-ray CCDs", Proc. SPIE, 4012, 720-730, 2000.

7 G.Y. Prigozhin, S.E. Kissel, M.W. Bautz, C.E. Grant, B. LaMarr, R.F. Foster, \& G.R. Ricker, "Characterization of the radiation damage in the Chandra X-ray CCDs", Proc. SPIE, 4140, 123-134, 2000.

8 S.L. O'Dell, M.W. Bautz, W.C. Blackwell Jr., Y.M Butt, R.A. Cameron, R.F. Elsner, M.S. Gussenhoven, J.J. Kolodziejczak, J.I. Minow, R.M. Suggs, D.A. Swartz, A.F. Tennant, S.N. Virani, \& K.M. Warren, "Radiation environment of the Chandra X-Ray Observatory", Proc. SPIE, 4140, 99-110, 2000.

J.J. Kolodziejczak, R.F. Elsner, R.A. Austin, \& S.L. O'Dell, "Ion transmission to the focal plane of the Chandra Xray Observatory", Proc. SPIE, 4140, 135-143, 2000.

S.L. O'Dell, W.C. Blackwell Jr,, R.A. Cameron, J.I. Minow, D.C. Morris, B.J Spitzbart, D.A. Swartz, S.N. Virani, \& S.J. Wolk, "Managing radiation degradation of CCDs on the Chandra X-ray Observatory", Proc. SPIE, 4851, 77-88, 2003. (Paper I)

http://www. spenvis.oma.be/spenvis/

12 E.C. Stone, A.M. Frandsen, R.A. Mewaldt, E.R. Christian, D. Margolies, J.F. Ormes, \& F. Snow, "The Advanced Composition Explorer", Space Science Rev., 86, 1-22, 1998.

E.C. Stone, C.M.S. Cohen, W.R. Cook, A.C. Cummings, B. Gauld, B. Kecman, R.A. Leske, R.A. Mewaldt, M.R. Thayer, B.L. Dougherty, R.I. Grumm, B.D. Milliken, R.G. Radocinski, M.E. Wiedenbeck, E.R. Christian, S. Shuman, \& T.T. von Rosenvinge, "The Solar Isotope Spectrometer for the Advanced Composition Explorer", Space Science Rev., 86, 357-408, 1998.

R.E. Gold, S.M. Krimigis, S.E. Hawkins III, D.K. Haggerty, D.A. Lohr, E. Fiore, T.P. Armstrong, G. Holland, \& L.J. Lanzerotti, "Electron, Proton and Alpha Monitor on the Advanced Composition Explorer spacecraft", Space Science Rev., 86, 541-562, 1998. "Modeling the Chandra space environment", Proc. SPIE, 4140, 111-122, 2000.

R. Müller-Mellin, H. Kunow, V. Fleissner, E. Pehlke, E. Rode, N. Roschmann, C. Scharmberg, H. Sierks, P. Rusznyak, I.E.S. McKenna-Lawlor, J. Sequeiros, D. Meziat, S. Sanchez, J. Medina, L. der Peral, M. Witte, R. Marsden, \& J. Henrion, "COSTEP comprehensive suprathermal and energetic particle analyzer", Solar Phys., 162, 483-504, 1995. Chandra x-ray CCDs", Proc. SPIE, 5898, [Paper 27], 2005.

C.E. Grant, G.Y. Prigozhin, B. LaMarr, \& M.W. Bautz, "Sacrificial charge and the spectral resolution performance of the Chandra advanced CCD imaging spectrometer", Proc. SPIE, 4851, 140-148, 2003.

C.E. Grant, M.W. Bautz, S.E. Kissel, \& B. LaMarr, "A charge transfer inefficiency correction model for the Chandra advanced CCD imaging spectrometer”, Proc. SPIE, 5501, 177-188, 2004. 
20 L.K. Townsley, P.S. Broos, J.A Nousek, \& G.P. Garmire, G. P., "Modeling charge transfer inefficiency in the Chandra Advanced CCD Imaging Spectrometer", Nucl Instruments Methods Phys. Res. A, 486, 751-784, 2002.

21 P.P. Plucinsky, N.S. Schulz, H.L. Marshall, C.E. Grant, G. Chartas, D. Sanwal, M. Teter, A.A. Vikhlinin, R.J. Edgar, M.W. Wise, G.E. Allen, S.N. Virani, J.M. DePasquale, \& M.T. Raley, "Flight spectral response of the ACIS instrument", Proc. SPIE, 4851, 89-100, 2003.

22 D.J. Williams, R.W. McEntire, C. Schlemm II, A.T.Y. Lui, G. Gloeckler, S.P. Christon, \& F. Gliem, "Geotail Energetic Particles and Ion Composition instrument", J. Geomag. Geoelect., 46, 39-57, 1994.

23 H. Spence \& J. Blake, "First observations by the CEPPAD imaging proton spectrometer aboard Polar", Advances in Space Res., 20, 933-936, 1998.

24

D.J. McComas, S.J. Bame, P. Barker, W.C. Feldman, J.L. Phillips, P. Riley, \& J.W. Griffee, "Solar-Wind Electron Proton Alpha Monitor (SWEPAM) for the Advanced Composition Explorer", Space Science Rev., 86, 563-612, 1998.

25 R.D. Zwickl, K.A. Doggett, S. Sahm, W.P. Barrett, R.N. Grubb, T.R. Detman, V.J. Raben, C.W. Smith, P. Riley, R.E. Gold, R.A. Mewaldt, \& S. Maruyama, "The NOAA Real-Time Solar-Wind (RTSW) system using ACE data", Space Science Rev., 86, 633-648, 1998.

${ }^{26}$ K.A. Costello, "Moving the Rice MSFM into real-time forecast mode using solar-wind driven forecast models", Ph. D. dissertation, Rice University, June 1997. 

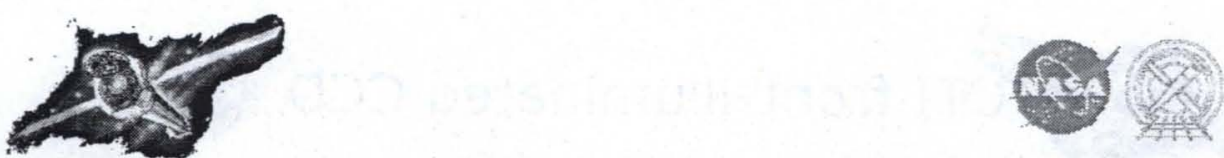

\title{
Managing radiation degradation of CCDs on the Chandra X-ray Observatory-III
}

\author{
Steve O'Dell, Tom Aldcroft, Bill Blackwell, Sabina Bucher, \\ Jon Chappell, Joe DePasquale, Catherine Grant, Mike Juda, \\ Eric Martin, Joe Minow, Steve Murray, Paul Plucinsky, \\ Dan Schwartz, Dan Shropshire, Brad Spitzbart, \\ Paul R. Viens, \& Scott Wolk \\ NASA Marshall Space Flight Center \\ Harvard-Smithsonian Center for Astrophysics \\ Jacobs Sverdrup \\ Northrop Grumman Space Technology \\ Massachusetts Institute of Technology Center for Space Research
}

Managing radiation degradation of CCDs on the Chandra X-ray Observatory-III

- Advanced CCD Imaging Spectrometer (ACIS) has 10 CCDs.

- ACIS-I comprises $2 \times 2$ CCD array; ACIS-S, $1 \times 6$ CCD array.

- 2 S CCDs are back-illuminated (BI); rest, front-illuminated (FI)

- During initial operations, ACIS FI CCDs experienced degradation.

- Charge-transfer inefficiency (CTI) increased dramatically.

- ACIS had been in focal plane during 8 radiation-belt passages.

- Trapped protons Rutherford scattered off $x$-ray mirror onto CCDs.

- Displacement damage increased FI-CCD CTI but not BI-CCD CTI.

- 45- $\mu \mathrm{m}$ Si shields BI charge-transfer channel for $\mathrm{E}_{\mathrm{p}}<2.2 \mathrm{MeV}$.

- 0.7- $\mu \mathrm{m}$ Si shields FI charge-transfer channel for $E_{p}<0.1 \mathrm{MeV}$.

- Radiation management substantially reduced rate of CTI increase.

- dCTI/dt now results from harder radiation outside proton belt.

- Strongly penetrating radiation contributes to CTI increase.

- Accounts for about 25\% FI CTI increase; nearly all BI CTI increase. 


\section{CTI front-illuminated CCD}

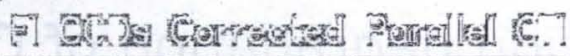

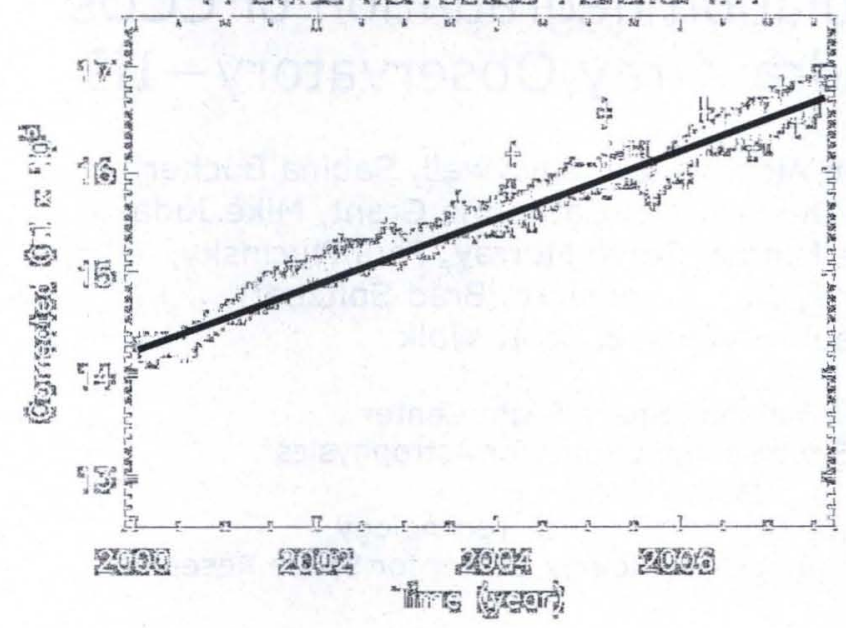

Managing radiation degradation of CCDs on the Chandra X-ray Observatory-III UV, X-Ray, and Gamma-Ray Space Instrumentation for Astronomy XV 2007 August 26-30, San Diego, CA USA
CTI measurement: $\mathrm{Fe}^{55} \mathrm{Mn}-\mathrm{K} \alpha(5.9 \mathrm{keV})$ ACIS $T_{F P}=-119.7^{\circ} \mathrm{C}$

Reference (2000 Jan):

$$
\text { CTI }=14.3 \times 10^{-5}
$$

Current (2007 July):

$$
\mathrm{CTI}=16.7 \times 10^{-5}
$$

Parallel average rate: $\mathrm{dCTI} / \mathrm{dt}=0.32 \times 10^{-5} / \mathrm{y}$

Framestore average rate: $\mathrm{dCTI} / \mathrm{dt}=0.062 \times 10^{-5} / \mathrm{y}$

MIT ACIS

SPIE Conference 6686 Paper 02
Slide 3

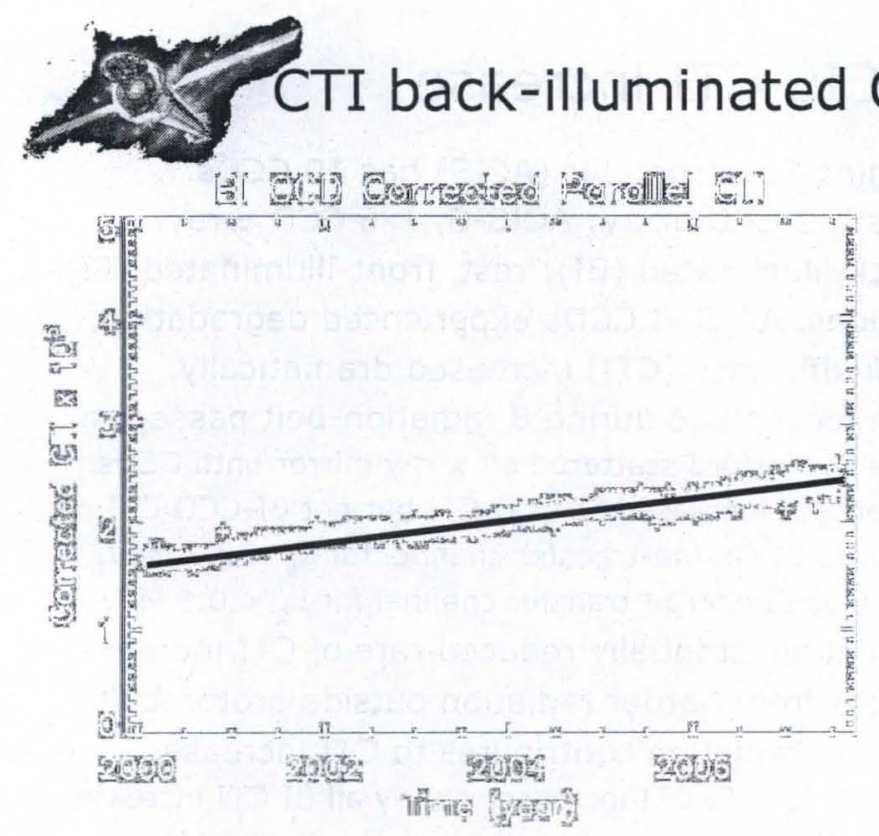

Managing radiation degradation of CCDs on the Chandra X-ray Observatory-III UV, X-Ray, and Gamma-Ray Space Instrumentation for Astronomy XV 2007 August 26-30, San Diego, CA USA

\footnotetext{
CTI measurement: $\mathrm{Fe}^{55} \mathrm{Mn}-\mathrm{K} \alpha(5.9 \mathrm{keV})$ ACIS T TPP $=-119.7^{\circ} \mathrm{C}$

Reference (2000 Jan): $\mathrm{CTI}=1.66 \times 10^{-5}$

Current (2007 July): $\mathrm{CTI}=2.40 \times 10^{-5}$
}

Parallel average rate: $\mathrm{dCTI} / \mathrm{dt}=0.097 \times 10^{-5} / \mathrm{y}$

Framestore average rate: $\mathrm{dCTI} / \mathrm{dt}=0.092 \times 10^{-5} / \mathrm{y}$

MIT ACIS

SPIE Conference 6686 Paper 02
Slide 4 


\section{Radiation protection}

- The Chandra X-ray Observatory is in a 63.5-h high elliptical orbit.

- 140-Mm-altitude (23- $\mathrm{R}_{\oplus}$ geocentric) initial apogee

- 10-Mm-altitude (3- $R_{\oplus}$ geocentric) initial perigee

- Orbit passes through proton and electron belts.

- Only real-time communication is during DSN contacts.

- Have nominally 3 DSN contacts/d for data dumps \& commanding.

- Command loads normally execute a 1-week observing plan.

- Revised operating procedures to protect ACIS against radiation.

- Use a radiation-protection configuration in high radiation.

- Translate ACIS into NIL position; power down video boards.

- Leave HRC in focal position; ramp down high voltage.

- Ensure radiation protection during all belt passages.

- Invoke radiation protection as needed for space weather.

- Employ autonomous, commanded, and scheduled protection.

Managing radiation degradation of CCDs on the Chandra X-ray Observatory-III

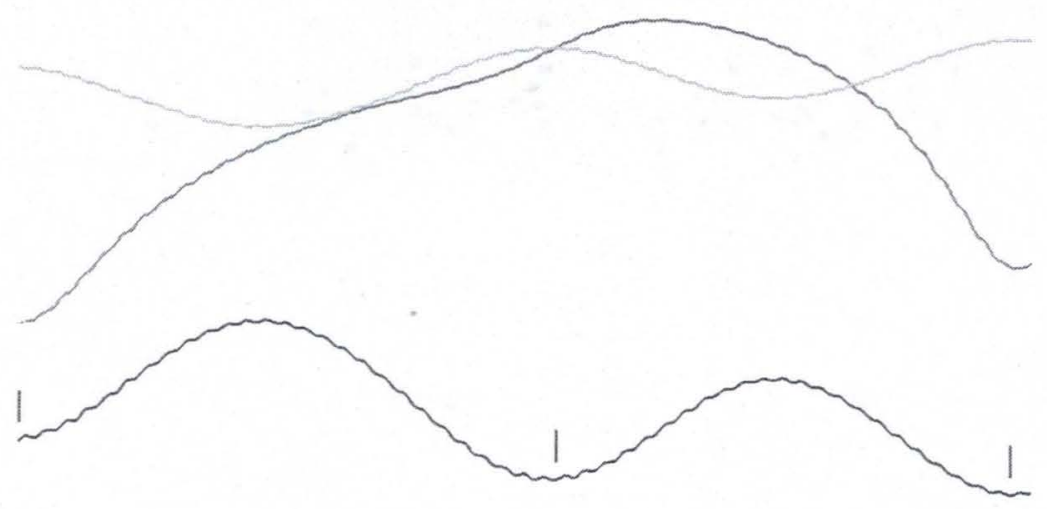

Managing radiation degradation of CCDs on the Chandra X-ray Observatory-III 


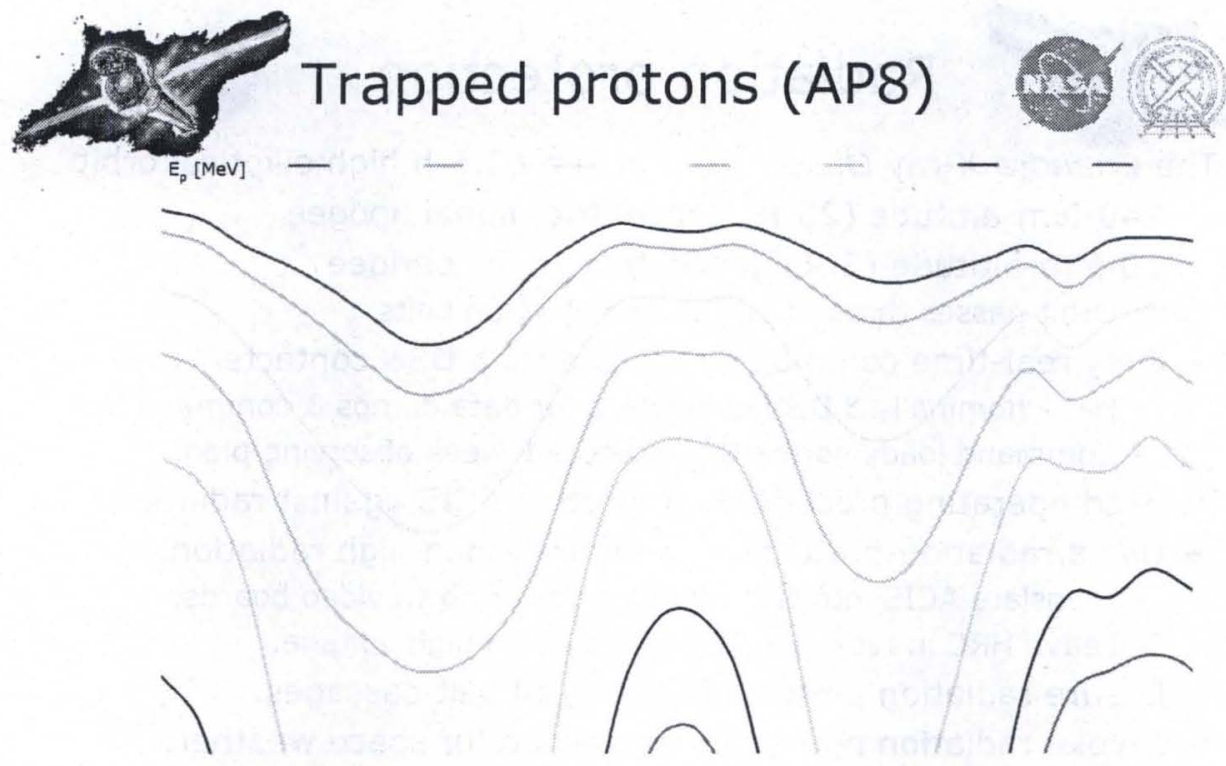

SPENVIS AP8

Managing radiation degradation of CCDs on the Chandra X-ray Observatory-III UV, X-Ray, and Gamma-Ray Space Instrumentation for Astronomy XV

SPIE Conference 6686 2007 August 26-30, San Diego, CA USA

Paper 02
Slide 7

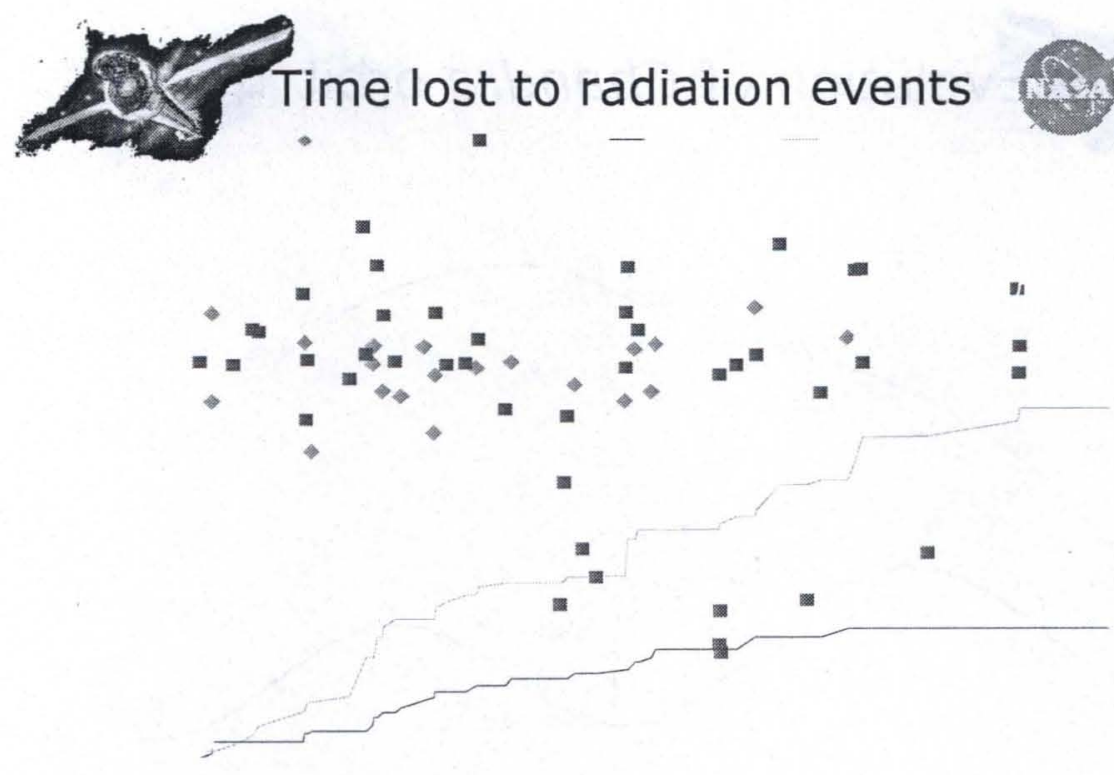




\section{Management strategy}

- Scheduled protection

- Move ACIS to NIL position for all radiation-belt passes.

- Pad AE8-predicted boundary against inaccuracies and variations.

- Manual intervention

- Monitor real-time ACE \& GOES data provided by NOAA's SEC.

- Monitor estimated soft-proton environment in Chandra's orbit.

- Built Chandra radiation model (CRM) of soft-proton environment.

- Use real-time solar proton and $\mathrm{Kp}$ data to drive data-based CRM.

- Manually protected 20 times for 1.911-Ms lost science.

- Autonomous protection

- EPHIN on Chandra measures electron \& $>5-\mathrm{MeV}$ proton flux.

- Lower-energy (0.1-1 MeV) protons cause most damage.

- Upon trigger, OBC halts load and moves ACIS to NIL position.

- Autonomously protected 44 times for 5.240-Ms lost science.

- Use EPHIN flux and CRM fluence to reduce pad on AE8 model.

- Recovers about 200 ks/y for science observations.
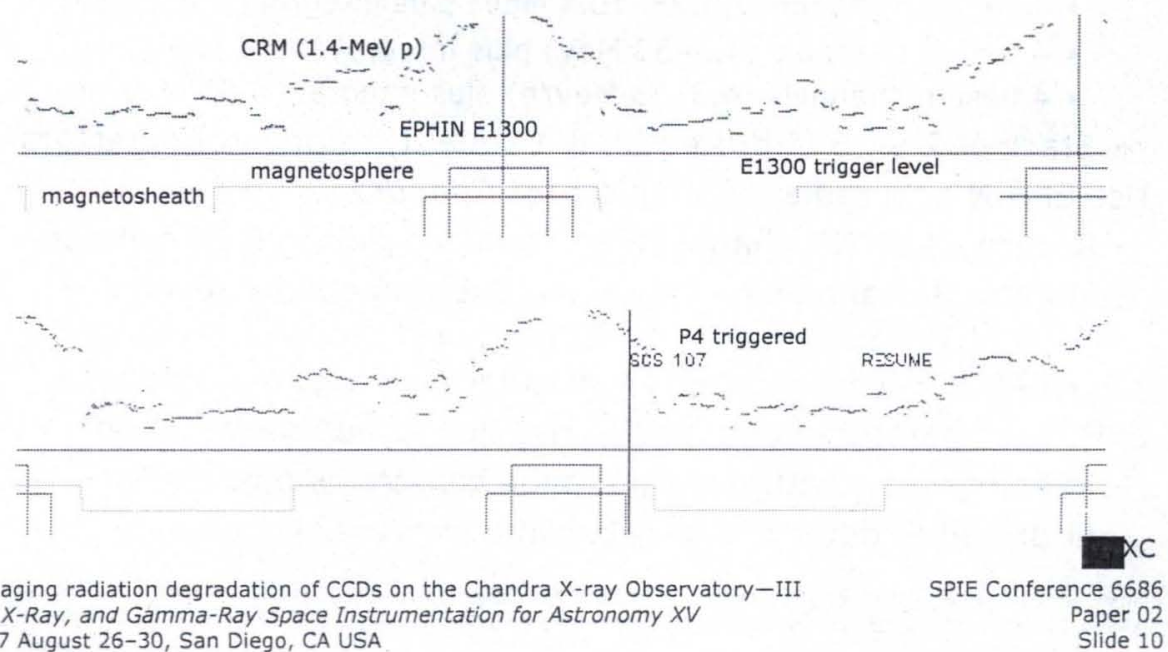


\section{Chandra radiation model}

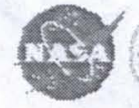

- Archival-data-based CRM

- Data through 2004

- 0.14-MeV protons

- Geotail EPIC

- Polar CEPPAD

- Streamline mapping

- GSM coordinates

- Correlated to $K_{p}$

- Magnetosphere [3]

- Magnetosheath [2]

- Solar wind [1]

- CRM proton flux

- Integrate past fluence
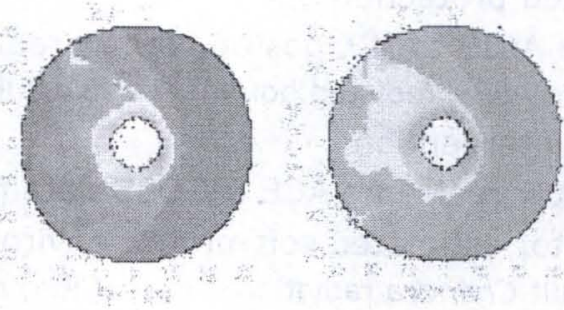

0.14-MeV p

- Project future fluence

Jacobs Sverdrup

SPIE Conference 6686
Paper 02

SPIE Conference 6686
Paper 02

Managing radiation degradation of CCDs on the Chandra X-ray Observatory-III UV, X-Ray, and Gamma-Ray Space Instrumentation for Astronomy XV 2007 August 26-30, San Diego, CA USA
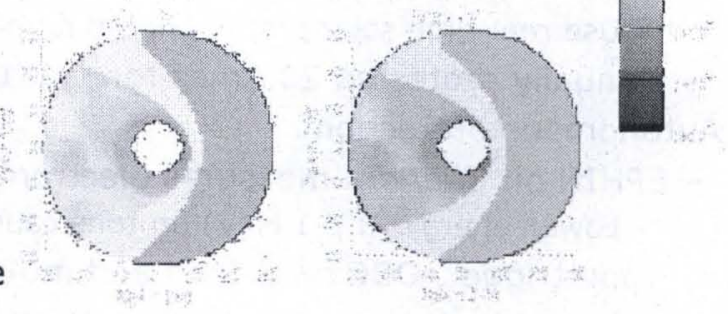
Slide 11

\section{EPHIN radiation monitor}

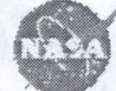

- Chandra EPHIN is SOHO COSTEP flight spare, from Univ. of Kiel.

- Electron, Proton, Helium Instrument (EPHIN)

- 4 electron channels (0.25-10.4 MeV) plus integral (> 8.7 MeV)

- 4 proton channels (4.3-53 MeV) plus integral (>53 MeV)

- 4 helium channels (4.3-53 MeV/n) plus integral (> $53 \mathrm{MeV} / \mathrm{n}$ )

- Stack of $2 \mathrm{Si}, 3 \mathrm{Li}$-drifted Si, \& 1 (guard) scintillator detectors.

- Use EPHIN as a radiation monitor for Chandra.

- Record all EPHIN channels for download during DSN contacts.

- Monitor 3 channels for triggering autonomous protection.

- P4 (4.3-7.8 MeV), P41 (41-53 MeV), and E1300 (2.6-6.2 MeV)

- RADMON process defends against rapid damage to instruments.

- EPHIN temperature continues to rise due to MLI degradation.

- Keeping temperature within range constrains operations.

- Li-drifted Si detectors may become irreversibly noisy. 


\section{EPHIN rail-voltage drop}

- Increased temperatures lead to current-limited state.

- Rail voltage drop causes drop in guard (G) detector high voltage.

- G-detector voltage drop results in reduced count rate.
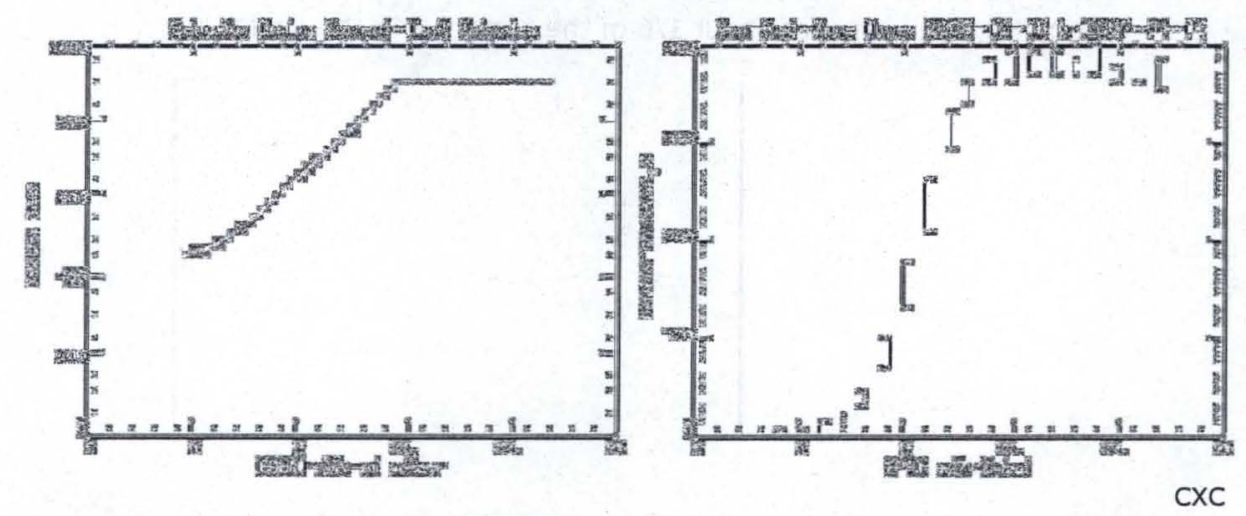

Managing radiation degradation of CCDs on the Chandra X-ray Observatory-III UV, X-Ray, and Gamma-Ray Space Instrumentation for Astronomy XV

SPIE Conference 6686 2007 August 26-30, San Diego, CA USA

\section{EPHIN noise increases}

- Rail voltage drop causes drop in guard (G) detector high voltage.

- G-detector voltage drop results in reduced count rate.

- G detector is used for anti-coincidence.

- Thus noise in other detectors increases during rail-voltage drops.

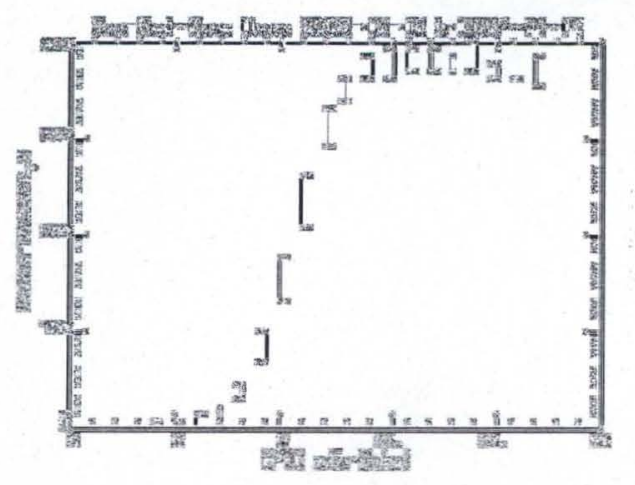

Managing radiation degradation of CCDs on the Chandra X-ray Observatory-III UV, X-Ray, and Gamma-Ray Space Instrumentation for Astronomy XV 
- Have patched flight software to replace EPHIN He data with HRC data.

- Facilitates potential use of HRC anti-co data by RADMON process.

- RADMON will use HRC anti-co data only if EPHIN channels are not useful.

- HRC anti-co rates correlate best with EPHIN P41 channel rates.

- HRC anti-co saturates at about $1 / 6$ of the current P41 threshold.

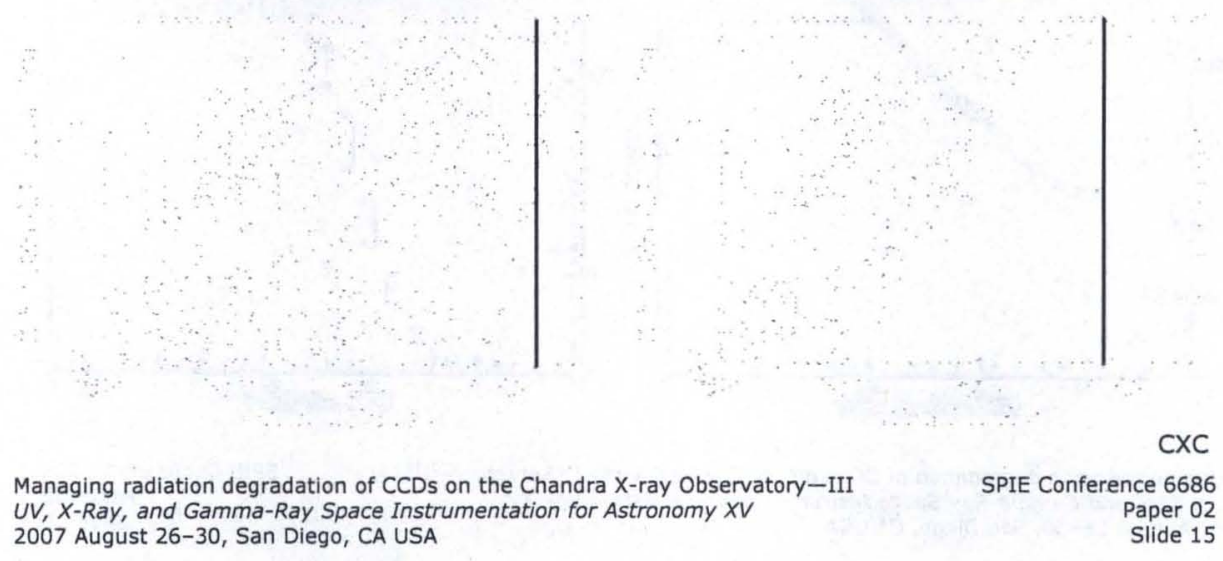

